\title{
Les arrêtés Parodi sur les salaires : un moment de la construction de la place de l'État dans le système français de relations professionnelles
}

Ministerial Texts and the specific History of Rules Production Process in French post-Liberation Industrials Relations

Jean Saglio

\section{(2) OpenEdition}

Journals

\section{Édition électronique}

URL : https://journals.openedition.org/travailemploi/4652

DOI : 10.4000/travailemploi.4652

ISSN : 1775-416X

Éditeur

DARES - Ministère du Travail

Édition imprimée

Date de publication : 30 juin 2007

Pagination : 53-73

ISSN : 0224-4365

Référence électronique

Jean Saglio, «Les arrêtés Parodi sur les salaires : un moment de la construction de la place de l'État dans le système français de relations professionnelles », Travail et Emploi [En ligne], 111 | juilletseptembre 2007, mis en ligne le 15 septembre 2009, consulté le 21 septembre 2021. URL : http:// journals.openedition.org/travailemploi/4652 ; DOI : https://doi.org/10.4000/travailemploi.4652 


\title{
Les arrêtés Parodi sur les salaires: un moment de la construction de la place de l'État dans le système français de relations professionnelles
}

\author{
Jean Saglio (*)
}

\begin{abstract}
Pour étudier le rôle de l'État et le mode de construction des branches professionnelles après guerre, l'article cerne les réactions des autres acteurs face à la régulation administrative centralisée mise en place en 1945, lorsque sont publiés les arrêtés Parodi Croizat fixant les salaires. En s'appuyant sur un corpus de textes réglementaires de l'année 1945 et sur le secteur du commerce alimentaire qui n'avait pas de régulation salariale formalisée avant la guerre, il soutient que ce schéma de fixation des salaires a structuré le mode de relations professionnelles en France en privilégiant la récrimination et la protestation et non la négociation et le dialogue.
\end{abstract}

«Un fonctionnaliste peut considérer comme allant de soi que, lorsqu'une société se donne une institution, elle se donne en même temps comme possédables toutes les relations symboliques et rationnelles que cette institution porte ou engendre - ou qu'en tout cas, il ne saurait y avoir de contradiction ou d'incohérence entre les «fins» fonctionnelles de l'institution et les effets de son fonctionnement réel, que chaque fois qu'une règle est posée, la cohérence de chacune de ses conséquences innombrables avec l'ensemble des autres règles déjà existantes et avec les fins consciemment ou «objectivement» poursuivies est garantie. Il suffit d'énoncer clairement ce postulat pour en constater l'absurdité; il signifie que l'esprit absolu préside à la naissance ou à la modification de chaque institution qui apparaît dans l'histoire (qu'on l'imagine présent dans la tête de ceux qui créent l'institution ou caché dans la force des choses ne change rien à l'affaire)» (CASTORIADIS, 1975, p. 170).

La Libération est une période charnière pour la structuration des relations professionnellesenFrance. Particulièrement sur les questions qui concernent la régulation des salaires. Avant cette période, et sauf en temps de guerre, l'État n'intervient guère en ces matières; après, même si ce n'est pas d'une manière homogène, il intervient continûment. La période est exceptionnelle au sens où, formellement, jusqu'à la loi de février 1950, l'État est censé fixer les salaires ; les autres acteurs apprennent à jouer leur rôle dans une configuration où l'État outrepasse sa traditionnelle fonction d'arbitre et de contrôleur des règles de procédure et s'arroge la compétence de fixation des règles de fond. C'est encore une période clef

(*) CNRS - PACTE, université Pierre-Mendès-France, Jean. Saglio@upmf-grenoble.fr dans la mesure où les normes de salaires ainsi fixées - et notamment les hiérarchies salariales établies dans les classifications professionnelles - resteront des références stables pendant un temps fort long, et en tout état de cause largement supérieur à la durée de vie de la forme de la régulation(1) elle-même. C'est donc un moment important pour comprendre les impacts de l'action de l'État en matière de relations professionnelles en France.

L'analyse de l'action de l'État, et notamment de son effet structurant au sens où elle influence fortement les comportements et les stratégies des autres acteurs par la stabilisation des règles de procédure, consiste le plus souvent à rechercher comment ces autres acteurs se conforment aux injonctions exprimées par le législateur. On raisonne alors en suivant l'hypothèse, souvent implicite, que l'État assume un leadership - ou un rôle instituant - accepté dans la société française. Une autre configuration est cependant possible, où l'effet structurant de l'action de l'État se comprend comme le produit de l'opposition des autres acteurs à l'injonction étatique. Dans cette seconde ligne, nous allons ici retenir l'hypothèse que la place éminente des branches industrielles dans la régulation salariale, telle qu'elle est consacrée par la loi de février 1950, peut être vue, au moins pour une part, comme un moyen pour les autres acteurs de contourner la régulation par les professions que l'État cherchait à mettre en place.

(1) Nous employons ce terme au sens défini dans les travaux de Jean-Daniel Reynaud (Reynaud, 1989; De Terssac, 2003). Dans la lignée des analyses de relations professionnelles initiée par John T. Dunlop, l'analyse du corpus des règles formelles et celle du système d'acteurs et de leurs rapports de forces sont donc totalement complémentaires. 
Nombre des caractéristiques et des difficultés du système français de relations professionnelles après la Seconde Guerre mondiale sont souvent imputées à la rupture du consensus politique de la Libération. L'événement clef est alors recherché dans l'ordre politique, autour de la question de la participation des communistes au gouvernement. Dans un tel schéma d'analyse, les syndicalistes de la CGT ne font que suivre les injonctions qui leur viennent de la hiérarchie du Parti communiste. En examinant de plus près la mise en place de la régulation salariale dans l'année 1945 et les problèmes qui sont alors soulevés, on mettra l'accent ici sur une explication complémentaire, voire alternative, dans laquelle l'accent est mis au contraire sur les effets déstructurants de l'intervention étatique centralisatrice: en substituant la décision à la négociation collective, l'État renvoie ainsi le syndicat à privilégier la protestation et la récrimination plutôt que la régulation.

En matière de méthode, on a ici privilégié une approche très «externe» de l'action de l'État, en privilégiant l'étude des formes et des contenus de décisions plutôt que celle des justifications. Il ne s'agit pas de comprendre pourquoi l'acteur étatique fait ce qu'il fait et quelles justifications il donne à son action, mais plutôt d'insister sur les effets de ses actions et de ses décisions sur le comportement et les stratégies des autres acteurs. La principale source constituée ici est donc limitée aux seuls textes réglementaires publiés sur les questions de salaires, traitements et appointements au Journal officiel sous la signature du ministre du Travail et ne concernant pas les personnels du ministère luimême. On trouvera en annexe I une présentation rapide de ce corpus.

L'examen attentif de ce matériau nous amènera à constater que la première orientation politique mise en œuvre par le ministère du Travail jusqu'au milieu de l'année 1945 consiste pour une large part à minimiser le rôle des branches professionnelles au sens que ce terme a aujourd'hui. Les problèmes posés par une telle option centralisatrice du ministère vont donc apparaître dès le mois de juin 1945 et pousser les syndicats, et notamment la CGT à adopter vis-àvis de l'État et du ministère du Travail une attitude et une stratégie consistant à privilégier la récrimination plutôt que le consensus. On s'interrogera en conclusion pour savoir si cette configuration où l'action de l'État provoque l'opposition des autres partenaires est spécifique du domaine des salaires.

Pour étudier la mise en place de la régulation salariale dans les secteurs où celle-ci n'était pas formalisée avant-guerre, on a ici focalisé l'attention sur le secteur du commerce alimentaire (2). Celuici n'existe guère en tant que tel avant la Libération. Les «conventions collectives de travail», au moins jusqu'à la fin des années 1930, concernent le plus souvent les salaires des ouvriers; dans les secteurs des commerces et des services, la régulation des salaires par la négociation collective est exceptionnelle avant-guerre. Avant 1936, Pierre LAROQuE (1935) n'en signale l'existence que pour les ouvriers boulangers. Entre 1936 et la guerre, une régulation collective se forme dans certains secteurs dont les contours professionnels sont déjà précisés (comme le notariat), où la régulation de la concurrence économique entre firmes est coutumière (les banques) voire où il existe déjà une implantation syndicale non négligeable. Les secteurs du commerce, et notamment des commerces de détail sont parmi ceux dans lesquels ces régulations de relations professionnelles sont les plus rares(3). On suivra dans ce cas l'action du ministère du Travail à partir des documents existants dans le dossier correspondant du fond Raffalovitch déposé au Centre des archives contemporaines de Fontainebleau (4).

\section{La procédure mise en place}

La régulation salariale mise en place à la Libération reste organisée selon la procédure prévue pour l'économie de guerre. Les salaires sont «déterminés, en principe, par voie administrative» (SELLIER, 1961, p. 63) et centralisée, c'est-àdire qu'ils sont fixés dans des textes signés par le ministre du Travail. Il ne s'agit cependant pas d'une détermination précise et complète des rémunérations par le ministère. Les entreprises conservent une certaine marge de manœuvre, notamment dans la fixation des salaires individuels: pour la plupart des situations et des emplois, ce qui est déterminé par la décision ministérielle n'est en effet pas le salaire effectif, mais la fourchette dans laquelle doit s'inscrire le salaire moyen de la catégorie. De plus, les taux ainsi fixés concernent les salaires horaires, et l'on doit garder en mémoire que bon nombre d'ouvriers sont encore "payés aux pièces» selon des systèmes variés pour lesquels le taux de salaire

(2) Nous avons utilisé pour ce faire les travaux et les matériaux de terrain accumulés lors du travail avec Sophie Le Corre: Le ministère du Travail et les secteurs tertiaires: la régulation des relations professionnelles dans le secteur du grand commerce alimentaire de 1950 à nos jours, dans le cadre des travaux menés à l'occasion du centenaire du ministère du Travail. C'est l'excellente connaissance que Sophie Le Corre a de ce secteur et de son histoire qui nous a permis de mieux comprendre les documents et les contextes de la branche.

(3) Sans être totalement inexistante, notamment au niveau local comme le remarque Anne Sophie BEau (2001) dont le travail porte sur une entreprise de commerce non-alimentaire. (4) CAC 19760121 - article 209 dans lequel on trouve notamment les rapports de Mademoiselle Deguy, inspectrice du travail en charge de suivre la négociation dans ce secteur ainsi que certains des accords signés entre patrons et syndicats. 
n'est que l'un des éléments de la fixation des «tarifs de pièces », mais non des revenus salariaux.

Dans un premier temps, les ordonnances d'aoûtseptembre 1944 majorent les salaires minima et les salaires réels (effectifs), mais décident que «le taux moyen d'une catégorie professionnelle ne peut dépasser le taux minimum de cette catégorie de $20 \% »$ (SELLIER, 1984, p. 187). Une remise en ordre de plus grande ampleur est cependant souhaitée. Elle sera mise en œuvre au cours de l'année 1945 par Alexandre Parodi, gaulliste et ministre du Travail, et poursuivie par son successeur Ambroise Croizat, leader de la métallurgie parisienne CGT, ministre communiste du travail du 21 novembre 1945 jusqu'à la crise gouvernementale de mai 1947. On focalisera ici l'attention sur la période de mars à novembre 1945, où se met en place cette régulation nouvelle.

À la différence de la période vichyste, un avis est demandé avant la décision à des «commissions techniques» comprenant des représentants des salariés et des employeurs. François Sellier, en comparant les deux périodes, parle ainsi de «régulation autoritaire» pour la période de Vichy et de «régulation souple» pour la période de l'après libération. Il manifeste toutefois quelques réticences quant à la représentativité des «commissions techniques» en remarquant qu'elles «comprenaient surtout des fonctionnaires et des membres de grands corps de l'État». Deux représentants «patronaux» et «ouvriers», sans autre précision, complétaient ces commissions. Le caractère centralisé s'associait ainsi au caractère économiquement abstrait de ces organes de consultation» (ibidem). Quoi qu'il en soit du caractère plus ou moins démocratique de cette consultation, la procédure retenue permet de produire, dans un laps de temps relativement court, un ensemble de règles salariales définissant pour ce qui concerne les emplois privés un «ordre des salaires » dont on peut penser qu'il est relativement cohérent. Les «arrêtés Parodi Croizat» resteront ainsi longtemps des références marquantes, notamment en matière de classification.

La lecture de l'ensemble des textes ministériels publiés au Journal officiel sur cette question des salaires dans les entreprises privées permet d'identifier une procédure commune de production de la réglementation. On peut en effet distinguer trois catégories de textes: en premier lieu des arrêtés ministériels que nous nommerons «primaires » qui définissent les champs professionnels et les catégories de classement. En second lieu des «décisions » de classification qui, comme leur nom l'indique, opèrent le classement des différents emplois dans ces catégories. Viennent ensuite - du moins logiquement - les différents textes modificatifs, arrêtés et le plus souvent décisions qui complètent et transforment ces règles antérieures. Pour chaque «régulation de branche» la succession de ces textes est respectée. Dans l'ensemble cependant, la publication des arrêtés et celles des décisions, voire même celle des textes complémentaires se chevauchent nettement. En analysant ces trois types de textes, nous pourrons donc percevoir comment la logique de l'action étatique parvient ou non à s'imposer et provoque le positionnement stratégique des autres acteurs du système.

\section{Les arrêtés primaires}

Dans l'image qu'on en garde le plus souvent, les arrêtés Parodi Croizat sont établis pour des branches professionnelles et correspondent à la politique du ministère du Travail. À examiner de plus près la collection de ces textes pour 1945, on constate que le débat de l'époque sur la définition de ce niveau de régulation est ouvert et notamment que la notion de branche professionnelle telle qu'elle sera pratiquée par la suite ne s'impose pas sans débat. On peut ainsi constater que, y compris sur la question des hiérarchies salariales, les formes de régulation qui sont retenues ne sont pas nécessairement celles qu'aurait souhaitées le ministère du Travail.

\section{Catégories génériques et champs d'application}

Le premier temps de la procédure est consacré à la détermination du cadre de négociation et à la fixation des catégories générales qui vont permettre les négociations et les décisions ultérieures. Il s'agit en premier lieu de délimiter ce qui sera nommé par la suite «champ d'application professionnel» c'est-àdire l'espace de régulation dans lequel le texte a vocation à s'appliquer. Il faut ensuite fixer les échelles de salaires, en définissant et hiérarchisant des catégories génériques, dans lesquelles vont s'inscrire les salaires de postes réels. Pour chaque «industrie», cette première période se termine par la publication au Journal officiel d'un arrêté, signé du ministre. On qualifie ici ce premier arrêté de «primaire» pour le distinguer des éventuels textes rectificatifs et modificatifs publiés ultérieurement. En matière de définition du champ d'application cette série de textes n'est pas homogène. Il y a bien des arrêtés « de branche» au sens où l'on entend aujourd'hui ce terme mais on trouve également deux autres catégories: des arrêtés interprofessionnels et d'autres dont le champ est chevauchant.

\section{Des arrêtés «de branche»}

La structure et les contenus de ces arrêtés sont homogènes et très tôt fixés: le canevas sur lequel sont construits les arrêtés et les contenus des règles ne varient guère. Du moins pour ce qui concerne ce que tout un chacun convient d'appeler les «salaires», c'est-à-dire quand il s'agit de fixer les rémunérations des ouvriers dont la spécialité profes- 
sionnelle correspond à une branche industrielle bien définie. L'encadré 1 présente le contenu des différents articles de l'arrêté concernant le bâtiment et les travaux publics de la région parisienne. Il s'agit chronologiquement du second arrêté publié dans la série (5). Il reprend, quoique dans un ordre légèrement différent les thèmes et les règles énoncés dans l'arrêté du 11 avril concernant la métallurgie. La principale différence est cependant qu'il comprend un article de moins: celui stipulant les modalités de calcul du «salaire au rendement». On peut donc retenir que, dès ce moment, la forme de ces arrêtés primaires est bien fixée et restera stable.

La longueur du texte varie plus nettement, notam-

Encadré 1
Contenu des articles de l'arrêté
salaires dans les industries
du bâtiment et des travaux publics
de la région parisienne (17 avril 1945)

Article 1: objet: les salaires des ouvriers dans les entreprises non artisanales.

Article 2: champ d'application.

Article 3: textes applicables pour la détermination des zones de salaires.

Article 4: définition des catégories ouvrières (cinq pour le BTP).

Article 5: salaires horaires par catégories, par échelon et par zones.

Article 6: définition des primes et bonifications, salaires des handicapés.

Article 7: modalités de l'égalité salariale hommes/ femmes.

Article 8: salaires des jeunes ouvriers et des apprentis.

Article 9: salaires des ouvriers dont les salaires antérieurs étaient supérieurs au barème.

Article 10: sanctions.

Article 11: entrée en vigueur au 15 mars 1945.

Article 12: désignation du directeur du travail pour l'application de l'arrêté.

ment du fait que l'énoncé des catégories génériques peut être tout à fait succinct ou plus détaillé. L'arrêté du BTP peut être pris pour exemple de la définition la plus succincte des catégories: la plus longue y est celle des «ouvriers hautement qualifiés» qui se limite à ces trois mots. Une plus grande complexité peut être observée dans d'autres définitions, comme par exemple celles de l'arrêté du 13 juillet sur les «salaires dans les industries graphiques» où les différences entre échelons dans les catégories d'ouvriers qualifiés sont soigneusement définies et où la qualification d'ouvrier hautement qualifié renvoie à plusieurs critères concomitants, portant tout à la fois sur les compétences de l'individu et sur le travail qu'il exécute. ( $c f$. encadré 2). Cette diversité conduit donc à supposer que le ministère du Travail n'a pas eu le pouvoir ou la volonté de prescrire en la matière une référence unique. Au contraire, il a utilisé des références professionnelles plus anciennes en se contentant de les rapporter à une grille qui a les apparences d'une grille unifiée.

L'opération d'élaboration de tels textes est relativement simple quand une régulation effective préexistait, en particulier quand il y avait eu, après mai 1936, des conventions collectives. On comprend donc aisément que les premiers textes sortis soient effectivement ceux concernant des branches dont l'existence était bien connue et dont les frontières étaient fixées: la métallurgie parisienne, le secteur du bâtiment et des travaux publics de la région parisienne sont les premiers secteurs pour lesquels le ministère publie, dès avril 1945 un arrêté (6). Le texte de l'arrêté de la métallurgie parisienne reprend le champ d'application et des définitions de catégories professionnelles qui avaient été produits dans les négociations antérieures, entre 1936 et 1940 ( $c f$. SAGLIO, 1986).

Il est cependant des cas où la procédure employée est différente. Ce qui amène à constater que la conception même du niveau de cette régulation intermédiaire est alors mal fixée. Au lieu que ce niveau soit celui de la branche industrielle, comme on en a pris la coutume ensuite, et se définisse par l'ensemble des salariés des entreprises dont la production relève de la branche en question, les champs de compétence des arrêtés Parodi relèvent encore de deux autres modes de définition. Certains vont réguler un ensemble d'emplois que l'on peut retrouver dans des entreprises produisant des biens ou des services très différents et donc relevant de plusieurs «industries»: conformément à un usage attesté dès l'époque, on va les nommer comme ayant un champ d'application «interprofessionnel»; d'autres vont utiliser, dans des cas semblables, une autre technique consistant à rattacher la régulation des salariés en question à l' «industrie» qui serait celle de leur rattachement principal.
(5) Si l'on examine les textes visés en référence et les techniques de décision, l'arrêté du 31 mars 1945 portant sur les «Salaires des employés de banque» correspond au modèle antérieur. Il apparaît tout à fait pertinent donc de se conformer à la coutume qui retient l'arrêté de la métallurgie parisienne, daté du 11 avril 1945, comme point de départ de cette série.
(6) «Salaires dans l'industrie des métaux de la région parisienne» signé le 11 avril et publié le 12 ; «Salaires dans les industries du bâtiment et des travaux publics de la région parisienne» signé le 17 et publié le 19 avril. 


\section{Les définitions des catégories génériques dans les arrêtés du BTP (17 avril) et des industries graphiques (13 juillet)}

\section{Industries du bâtiment et des travaux publics}

«Article 4. - les ouvriers des industries du bâtiment et des travaux publics sont répartis dans les catégories professionnelles ci-après:

$1^{\text {re }}$ catégorie: manœuvre ordinaire;

$2^{\mathrm{e}}$ catégorie: manœuvre spécialisé;

$3^{e}$ catégorie: ouvriers spécialisés, cette catégorie comprenant de un à trois échelons;

$4^{\mathrm{e}}$ catégorie: ouvriers qualifiés, cette catégorie comprenant trois échelons;

$5^{\mathrm{e}}$ catégorie: ouvriers hautement qualifiés ».

\section{Industries graphiques} après:

«Article. - les ouvriers des industries graphiques sont répartis dans les catégories professionnelles définies ci-

Première catégorie: manœuvres:

- $1^{\text {er }}$ échelon: ouvriers exécutant des travaux simples qui n'entrent pas dans le cycle des fabrications, qui n'exigent ni connaissance professionnelle, ni force physique supérieure à la moyenne (nettoyages, charrois, manutentions) ;

$-2^{e}$ échelon: manœuvres de force exécutant sans initiation professionnelle préalable des travaux simples qui exigent un effort physique et manœuvre exécutant des travaux qui, sans nécessiter aucune connaissance professionnelle, comportent une initiation de courte durée.

Deuxième catégorie: ouvriers spécialisés:

$1^{\mathrm{er}}$ échelon: ouvriers légèrement spécialisés exécutant des travaux peu variés qui ne nécessitent que des connaissances professionnelles limitées;

$2^{\mathrm{e}}$ échelon: ouvriers spécialisés connaissant une partie seulement d'un métier qualifié faisant l'objet d'un certificat d'aptitude professionnelle ou ne possédant pas l'habileté et le rendement exigés des ouvriers qualifiés.

Troisième catégorie:ouvriers qualifiés:

$1^{\text {er }}$ échelon: ouvriers qualifiés connaissant complètement un métier dont l'apprentissage est codifié et sanctionné par un certificat d'aptitude professionnelle qui, après une période de perfectionnement, ont acquis l'habileté correspondant à un rendement normal déterminé par les usages professionnels et prévu au certificat d'aptitude professionnelle. Ces connaissances et cette habileté pouvant provisoirement résulter d'une longue pratique du métier;

$2^{\mathrm{e}}$ échelon: ouvriers qualifiés qui ont des connaissances spéciales, ou plusieurs qualifications, ou qui sont chargés d'une manière habituelle de travaux pénibles ou peu courants;

$3^{e}$ échelon: ouvriers qualifiés qui sont chargés habituellement de la conception et de l'exécution de leur travail ou qui exécutent régulièrement des travaux difficiles et de technique moderne, ou qui sont responsables de la conduite et de l'entretien d'une machine moderne à grand rendement et de grand format.

Quatrième catégorie: ouvriers hautement qualifiés:

- ouvriers d'élite de qualification exceptionnelle, soit technique soit artistique, exerçant avec un excellent rendement et d'une manière continue un métier de technique moderne encore en évolution. Ils remplissent leurs fonctions sans aide et sans conseils. Ils connaissent les métiers voisins ».

\section{Des arrêtés dont le champ est interprofessionnel}

Les conventions collectives antérieures à 1936 étaient dans leur grande majorité des «conventions collectives de travail» et ne concernaient donc que le travail ouvrier(7). La branche conventionnelle,

(7) De la même manière, le «droit du travail» est fréquemment, avant la Seconde Guerre mondiale le «droit ouvrier». Par exemple: Scelle Georges (1922), Le droit ouvrier, Paris, Armand Colin. À côté, et différents, étaient les mondes des employés, et surtout celui des différents «collaborateurs». Si les rémunérations des ouvriers étaient bien des «salaires», les autres catégories percevaient des «traitements», «émoluments» ou autres «appointements». ou plutôt - selon la terminologie en usage à l'époque - «l'industrie», est donc l'espace de régulation des salaires, c'est-à-dire pour l'essentiel des salaires ouvriers. Dans le mouvement de 1936, on verra apparaître des conventions collectives ou des avenants spécifiques concernant d'autres catégories. Anne-Sophie BEAU (2001) repère ainsi le cas d'une convention collective des dactylographes et sténographes signée en 1936 dans la région lyonnaise qui aurait un champ d'application interprofessionnel. Mais ce mouvement n'a rien de systématique.

Dans le cadre des arrêtés Parodi, on va observer un mouvement tendant à instaurer des régulations interprofessionnelles pour certains métiers et profes- 
sions. Le 16 mai le ministre signe ainsi un arrêté intitulé « Salaires des employés de bureau et services annexes dont la fonction présente un caractère interprofessionnel, occupés dans les établissements industriels, commerciaux, les professions libérales, les offices publics et ministériels, les syndicats, sociétés civiles et associations de quelle que nature que ce soit» et la décision de classification correspondante est datée du même jour. L'article 2 de cet arrêté ( $c f$. encadré 3 ) énonce les six catégories qui doivent être utilisées pour les classifications.

\section{Encadré 3}

\section{Arrêté du 16 mai 1945: catégories de classement des employés et personnels de service}

«Article 2. - les employés visés à l'article précédent sont répartis dans les catégories définies ciaprès:

A) Travailleurs manuels et personnel de service:

- catégorie I. - travailleurs effectuant exclusivement des travaux manuels simples et courants n'exigeant ni aptitudes particulières ni connaissances spéciales et qui n'entrent pas dans le cadre particulier des activités de l'entreprise (coefficient: 100);

- catégorie II. - travailleurs répondant à la définition de la catégorie I, mais que leur activité met en contact avec le public (coefficient: 110).

B) Employés:

- catégorie I. - employés effectuant des travaux qui n'exigent qu'une initiation de courte durée ou une formation professionnelle très simple ( $1^{\text {er }}$ échelon: coefficient 110; $2^{\mathrm{e}}$ échelon: coefficient 120 );

- catégorie II. - employés ayant des connaissances professionnelles et une expérience du métier qui ne peuvent être acquises que par un apprentissage ou une pratique suffisante (1 ${ }^{\mathrm{er}}$ échelon: coefficient $125 ; 2^{\mathrm{e}}$ échelon: coefficient 135$)$;

- catégorie III. - employés exécutant des travaux qui exigent une formation professionnelle approfondie (coefficient 150);

- catégorie IV. - employés hautement qualifiés par leur compétence professionnelle, qui, pour l'exécution des travaux qui leur sont confiés, sont appelés à prendre des initiatives et des responsabilités (coefficient 170) ».

La même définition du champ interprofessionnel est reprise dans un arrêté du 21 juillet qui «a pour objet de fixer les salaires du personnel masculin et féminin travaillant dans les cantines ». Lequel arrêté énonce directement la classification et les salaires des emplois concernés. L'arrêté du 15 octobre, fixant les appointements des «assistantes sociales du travail» se définit pour champ d'application «les établissements industriels et commerciaux». De la même façon, mais plus tardivement (31 janvier 1946), l'arrêté concernant les personnels des services médicaux et sociaux sera promulgué avec un champ d'application interprofessionnel.

Un arrêté du 4 juin fixant les «appointements des ingénieurs, techniciens, agents de maîtrise et agents des cadres supérieurs de toutes professions » a également un champ d'application interprofessionnel, mais son intitulé même fait savoir qu'il s'agit d'un «ajustement provisoire» et les arrêtés définitifs concernant ces emplois paraîtront à partir du quatrième trimestre et seront formulés selon les champs professionnels de branche.

\section{Des arrêtés dont le champ est chevauchant}

En cas de chevauchement, c'est-à-dire lorsqu'un emploi dans une entreprise ressort d'un métier considéré comme caractéristique d'une autre industrie, la doctrine semble être de considérer l'arrêté de cette autre industrie comme définissant les conditions minimales de salaires. Le principe est énoncé précisément dans l'arrêté du 7 juillet sur les salaires des ouvriers d'entretien: «Les ouvriers d'entretien qui n'exercent pas une spécialité particulière à l'industrie qui les emploie devront être, dans la classification relative à cette industrie, rangés dans des catégories ou des échelons leur assurant des conditions de rémunération qui ne pourront être inférieures à celles résultant des dispositions concernant leur profession d'origine.»

L'énoncé même de cette disposition conduit à une certaine perplexité que l'exemple suivant permet d'expliciter. Une catégorie d' « ouvriers d'entretien » qui apparaît à plusieurs reprises dans les différents textes est celle des opérateurs de centrales électriques d'usine. Les décisions de classification de la métallurgie du 17 juillet et du 6 octobre en font une sous-branche de la métallurgie. Les «conducteurs» «de compresseur», «de Diesel» ou «tableau et turbine» sont classés en assimilés professionnels de $1^{\text {er }}$ échelon; le «chauffeur breveté ou commissionné» et le «chef de chauffe» sont eux des ouvriers professionnels $1^{\text {er }}$ échelon; leurs salaires horaires minima sont donc de 28 francs l'heure, ce qui les place au coefficient 140. Par contre, la décision du 28 août pour les industries chimiques, dans la sous-branche des soudières, classe en «ouvrier qualifié» coefficient 135 le «machiniste à la centrale électrique (Dombasle)». Il semble donc que le travail de comparaison et d'alignement n'ait pas été effectué. N'est-ce pas l'indice que les négociateurs des industries chimiques n'ont pas accepté de respecter le principe et de s'aligner sur les résultats obtenus dans la métallurgie?

Quoi qu'il en soit de son effectivité, le simple énoncé de cette diversité fait comprendre la complexité de la réglementation qui se met en place. Dans une même entreprise, on pourrait ainsi avoir affaire avec trois types de textes différents, les premiers renvoyant à l'«industrie» de l'entreprise en question, les seconds renvoyant à une autre 
industrie et enfin les derniers étant spécifiques de certaines professions. Si la première définition de champ correspond effectivement à des institutions de représentation existantes, tant du côté des salariés que des employeurs, les deux autres soulèvent des problèmes difficiles quand on cherche à leur associer des institutions de représentation des acteurs de terrain: quelle institution syndicale, pour les salariés comme pour les employeurs, va être légitime pour engager ses mandants dans une négociation de salaires concernant l'ensemble des employés? L'initiative du ministère de faire exister des règles salariales interprofessionnelles est contradictoire avec la structure des acteurs dont les définitions privilégient les identités industrielles. On peut penser que, au moins pour une part, l'effort d'institution du groupe des cadres dont témoigne la création de la CFE - CGC en 1944 participe de cette tentative d'instaurer une régulation fondée sur d'autres bases que les classiques industries. Cette technique de catégorisation restera vivace dans la tradition des statisticiens pour définir les contours des groupes sociaux et des identités professionnelles $(8)$ mais - on va le constater - sera très vite abandonnée pour traiter effectivement des questions de régulations salariales.

\section{Niveaux de revenus et hiérarchies salariales}

Après le champ d'application, le second objet de l'arrêté primaire est la définition des hiérarchies salariales. Pour les ouvriers et les employés, cellesci sont énoncées en valeur du salaire horaire et accessoirement en coefficients. Le fondement de la hiérarchie est toujours le même et c'est la valeur de 20 francs l'heure correspondant au salaire horaire minimum du manœuvre ordinaire des industries des métaux. Les textes définissent, habituellement mais non systématiquement, un salaire horaire moyen maximum par catégorie. La fourchette de salaire est alors habituellement de l'ordre de 7,5 à $10 \%$ entre le minimum et ce «salaire moyen maximum ». La référence à des coefficients - la valeur 100 étant attribuée au «manœuvre ordinaire», «travailleur ordinaire», etc. - est fréquente mais non systématique. La rémunération est exprimée en valeur mensuelle pour les appointements des ingénieurs, cadres, techniciens et agents de maîtrise, mais aussi parfois pour les salaires des employés. La conversion se fait alors par la prise en compte de 173,5 heures travaillées dans le mois. La rémunération à la tâche, aux pièces ou au rendement est envisagée pour les ouvriers. Dans ce cas, il est prévu que les tarifs d'ouvrage soient établis de manière à permettre de dépasser les rémunérations minimales. Pour la métallurgie

(8) On peut rattacher à cette tradition aussi bien le travail de Luc Boltanski (1982) sur les cadres que celui d'Alain Desrosières et Laurent ThÉvenot (1988) sur les catégories socioprofessionnelles. parisienne, ce dépassement est fixé dans un premier temps à $20 \%$ du salaire minimum(9). Il est relevé à $25 \%$ dès le 24 avril. Un arrêté du 31 mai supprime tout simplement cette référence (10) et lui substitue une limitation par référence aux pratiques d'emploi qui est de fait incontrôlable.

Comme on le voit à travers l'évocation de ce seul exemple, les niveaux de salaires ne sont pas totalement fixes et définis au cours de l'année. Un taux annuel d'inflation proche de $40 \%$ rend en effet difficile de s'en tenir à une limitation stricte. Néanmoins, on peut considérer que les niveaux de rémunération observables dans les arrêtés signés par Alexandre Parodi sont comparables les uns aux autres du fait qu'ils prétendent tous - même ceux de la fin de l'automne - définir les salaires effectifs à la date du 15 mars.

Cette série d'arrêtés correspond-elle à une politique salariale cohérente du ministère ? On examinera les hiérarchies ainsi définies en analysant les règles portant sur les salaires les plus bas et celles portant sur les plus élevés. Pour éliminer les problèmes liés à la prise en compte de la dérive inflationniste, on se limitera dans ce premier temps à la prise en compte de textes signés avant le mois d'août.

\section{La base de la hiérarchie}

Le salaire horaire minimum du manœuvre de l'industrie des métaux de la région parisienne est bien le coefficient 100, mais il n'est pas le salaire de base de la hiérarchie salariale. Quatre types de critères permettent de justifier, tout à fait légalement, la rémunération du travail à des salaires inférieurs.

En premier lieu, les salaires varient en fonction de l'implantation des entreprises. Le salaire de 20 francs l'heure est celui du manœuvre dans les zones les mieux payées de la région parisienne. Il existe en effet quatre catégories de zones de salaires pour la seule région parisienne et le salaire minimum du même manœuvre homme n'est que de 15 francs l'heure dans la quatrième zone.

Le salaire minimum varie également en fonction du genre. La chose est parfois clairement affirmée, quand les catégories de classification sont différentes pour les femmes et pour les hommes, comme c'est le cas notamment dans les industries textiles ou celles du vêtement. Le principe de l'égalité homme femme apparaît bien affirmé dans d'autres textes,

(9) Article 7 de l'arrêté du 11 avril pour la métallurgie parisienne: «Pour les ouvriers travaillant au rendement, le salaire horaire moyen maximum est fixé à $120 \%$ du salaire horaire minimum.»

(10) Article 2: «Pour les ouvriers travaillant au rendement, le salaire moyen maximum est supprimé, sous réserve que les tarifs au rendement restent déterminés sur les bases de calcul normales pour chaque profession et que leur application n'ait pas pour objet ou pour effet une surenchère des salaires de nature à provoquer des débauchages de main-d'œuvre». 
mais il est parfois accompagné de précisions qui amènent à supposer qu'il n'a pas alors le sens que lui donnerait une lecture plus moderne. Ainsi dans l'arrêté de la métallurgie parisienne (11), l'article 8 rappelle bien le principe: "À conditions égales de travail et de rendement, le calcul de la rémunération doit être établi sur les mêmes bases pour les hommes et pour les femmes.» Mais le rédacteur fait suivre ce principe d'une précision dont l'existence montre que la discrimination reste parfaitement envisageable: «En aucun cas les taux minima des salaires des femmes ne pourront être inférieurs de plus de $10 \%$ aux taux minima des salaires des hommes de même catégorie professionnelle.»

Le troisième critère utilisable pour minimiser les taux de salaires est celui du handicap. L'article 5 de l'arrêté de la métallurgie parisienne donne en la matière la formulation que l'on retrouve à peu près inchangée dans l'ensemble de la série: «Lorsque le chef d'entreprise est appelé à occuper des ouvriers que leurs aptitudes physiques mettent dans une condition d'infériorité notoire sur les ouvriers de la même catégorie, il pourra, exceptionnellement, leur appliquer un salaire inférieur au salaire minimum. La réduction possible de salaire ne pourra excéder le dixième du salaire minimum. D'autre part, le nombre des ouvriers d'une catégorie auquel s'appliquera cette réduction ne pourra excéder le dixième du nombre d'ouvriers de la catégorie. Ces proportions peuvent être modifiées par décision spéciale de l'inspecteur divisionnaire du travail et de la main d'œuvre.»

Enfin le dernier argument permettant de justifier un salaire inférieur au salaire minimum du manœuvre est celui de l'âge. Indépendamment de toute situation d'apprentissage, les jeunes de moins de 18 ans voient les salaires qui leur sont dus amputés d'un certain quantum et l'arrêté de la métallurgie en exprime, une fois de plus, la norme telle qu'elle sera généralement reprise par les textes suivants: "Article 9. - les taux de salaires des jeunes ouvriers et ouvrières âgés de moins de dixhuit ans révolus sont fixés comme suit en fonction du salaire des ouvriers et ouvrières adultes de leur catégorie professionnelle:

$$
\begin{array}{ll}
\text {-De } 14 \text { à } 15 \text { ans... } & 50 \% . \\
\text {-De } 15 \text { à } 16 \text { ans.... } & 60 \% . \\
\text {-De } 16 \text { à } 17 \text { ans... } & 70 \% . \\
\text {-De } 17 \text { à } 18 \text { ans.... } & 80 \% » .
\end{array}
$$

On peut fort légitimement considérer que ces différents critères peuvent se cumuler. Une jeune femme de 16 à 17 ans non handicapée travaillant dans un établissement métallurgique de la quatrième zone de salaires de la région parisienne pourra donc voir son salaire minimum tourner autour des 10 francs l'heure.

\section{Le haut de la hiérarchie des salaires ouvriers et employés}

On a souvent tendance à considérer que la fixation du salaire des ouvriers qualifiés constitue l'un des pivots de la définition des hiérarchies salariales. On constate bien que tous les arrêtés concernant les ouvriers et les employés contiennent ainsi la référence à un «ouvrier», un «travailleur» ou un «employé» qualifiés. Dont l'écart de rémunération par rapport au manœuvre de base est contenu dans une fourchette relativement étroite: de $35 \%$ supérieur dans la chimie à 52,5\% dans le bâtiment.

Pour les plus hautes rémunérations ouvrières, les variations sont nettement plus importantes (12). Le salaire horaire moyen maximum de l'ouvrier le plus qualifié de la métallurgie parisienne est fixé à 36,55 francs dans l'arrêté du 11 avril. Dans le bâtiment et les travaux publics, le salaire de 35,70 est présenté comme étant tout à la fois le minimum et le maximum individuels. Ces deux arrêtés ne contiennent pas de références à des coefficients. Dans les industries du verre (arrêté du 19 mai) le coefficient maximum est de 200 et le salaire moyen maximum de 43 francs. Dans les industries des carrières et matériaux, qui semblent fonctionner en parallèle avec le BTP, le salaire de l'ouvrier hautement qualifié est de 39,25 , sans coefficient explicite, mais il s'agit d'un texte du 13 juillet.

Les salaires des employés sont définis par référence à des grilles similaires. Pour la plus haute catégorie «interprofessionnelle» d'employés, le salaire minimum est ainsi fixé au coefficient 170 et le salaire maximum au coefficient 200 par l'arrêté du 16 mai (article 7), et il s'agit cette fois d'un maximum individuel et non d'un salaire moyen de la catégorie. La décision de classification qui accompagne cet arrêté, signée le même jour permet de comprendre que c'est l'ensemble de la catégorie des employés qui est censé se tenir dans cet éventail de salaires. La quatrième - et plus haute - catégorie des employés comprend en effet quatre emplois dont les descriptions sont explicites:

«- comptables commerciaux: traduisent en comptabilité toutes les opérations commerciales et financières, les composent, les assemblent, pour pouvoir en tirer: prix de revient, balance, bilan, statistiques, prévision de trésorerie;

- comptables industriels traduisent en comptabilité les opérations industrielles (approvisionnements, fabrication, éventuellement immobilisation) en déduisent les prix de revient et donnent tous rensei-

(12) Pour ces observations, on ne prend en compte que les salaires de la première zone géographique.

(11) Dès la première version du 11 avril. 
gnements sur les prix de revient aux différents stades de la fabrication;

- secrétaires de direction collaborateurs immédiats du chef d'établissement qui doit(13) faire preuve d'initiative pour interpréter les instructions qui lui sont données et qui sont susceptibles d'avoir des responsabilités;

- traducteurs. »

Les arrêtés concernant les employés dans les diverses branches professionnelles s'inscrivent bien dans ces limites, du moins dans un premier temps. Ainsi la plus haute catégorie des employés de banque définie comme «employé hautement qualifié par leur compétence professionnelle, qui, pour l'exécution des travaux qui leur sont confiés, sont appelés à prendre des initiatives et des responsabilités et dont l'emploi peut être assimilé à ceux rangés dans la catégorie correspondante de l'article 2 de l'arrêté précité du 16 mai 1945» (14) est rémunérée au coefficient 170 . De même l'arrêté du 16 mai sert de référence dans les arrêtés du 17 mai pour le commerce non alimentaire et du 28 mai pour les ouvriers et employés des commerces de gros et de détail de l'alimentation.

Dans cette série des salaires et traitements des employés, certaines professions font cependant éclater le cadre prescrit. Ainsi les arrêtés parallèles, publiés le même jour et concernant l'un les pharmacies d'officine et l'autre les laboratoires d'analyse biologique définissent une catégorie $\mathrm{V}$ au coefficient 200. L'arrêté des pharmacies définit même une catégorie VI: «Personnel qui possède des qualités techniques ou commerciales au-dessus de la normale et assume l'exécution de travaux comportant une large initiative. Catégorie exceptionnelle» au coefficient 225. Il est vrai que ces deux textes sont signés le 16 juin et que l'on peut donc se demander si, à cette date, la politique ministérielle de résistance à l'ouverture de l'éventail de salaires est encore aussi nette.

\section{La reprise de régulations antérieures?}

Cette première phase de la procédure débouche donc sur la publication de «l'arrêté portant fixation des salaires». Elle peut être plus ou moins rapide. Le tableau 1 synthétise le calendrier de publication des arrêtés «primaires» sur l'année 1945 en distinguant selon les catégories professionnelles visées. On observe donc que la priorité semble bien être la signature des arrêtés concernant les ouvriers : sur les trente-sept arrêtés de cette catégorie, plus du tiers (quatorze) sont signés dès la fin mai et une moitié (dix-neuf) le sont avant la fin juin. Le rythme de signature des arrêtés concernant les employés est plus régulier dans l'année. Sur les vingt-cinq signés, quatre seulement le sont à la fin mai et les 50\% (treize) ne sont atteints qu'à la fin juillet. Quant aux ingénieurs et cadres, les arrêtés les concernant apparaissent seulement - et en très faible nombre - à l'automne 1945. Ce décalage des calendriers traduit encore l'influence de l'existence préalable des conventions collectives : c'est dans le monde ouvrier qu'elles étaient les plus courantes avant guerre, et il n'est donc pas étonnant de retrouver dans les premiers arrêtés concernant les mondes ouvriers ceux de la métallurgie, du BTP ou des industries chimiques qui avaient déjà une convention collective de travail avant 1940. Dans le monde des employés, les conventions signées étaient rares avant guerre, et il paraît difficile d'observer si l'existence d'une

Tableau 1

Rythme de publication des arrêtés et décisions de salaires en 1945

\begin{tabular}{|l|c|c|c|c|r|r|}
\hline & Ouvriers & Employés & $\begin{array}{c}\text { Ingénieurs } \\
\text { et cadres }\end{array}$ & Interprofessionnel & Arrêtés & Décisions \\
\hline Avril & 2 & 0 & & & 2 & \\
\hline Mai & 12 & 4 & & 1 & 17 & 4 \\
\hline Juin & 5 & 3 & & & 15 & 14 \\
\hline Juillet & 7 & 6 & & & 7 & 16 \\
\hline Août & 6 & 1 & & & 5 & 6 \\
\hline Septembre & 2 & 2 & 1 & & 6 & 17 \\
\hline Octobre & 2 & 3 & & & 6 & 6 \\
\hline Novembre & 1 & 4 & 1 & & 4 & 9 \\
\hline Décembre & 0 & 2 & 2 & $\mathbf{4}$ & $\mathbf{7 0}$ & $\mathbf{8 0}$ \\
\hline Total & $\mathbf{3 7}$ & $\mathbf{2 5}$ & $\mathbf{4}$ & & & \\
\hline
\end{tabular}

$N B$ : on n'a pris en compte dans ces données ni les textes qui sont de simples rectificatifs, ni ceux qui portent sur les questions de zones de salaires.

(13) Sic.

(14) Article 3 de l'arrêté du 18 mai. ancienne tradition de négociation a ou non accéléré le processus de production de la régulation. 
L'analyse du dossier concernant les commerces alimentaires permet de comprendre où sont les principales difficultés dans cette phase de la régulation. La première consiste à mettre autour de la table les employeurs et les représentants de salariés qui acceptent le principe de constituer une branche professionnelle - et qui ne relèvent pas d'une branche où cette régulation existe déjà (15). Les difficultés proviennent ici du monde patronal: la définition de l'acteur patronal est en effet synonyme de la définition de la branche. Le principe affiché pour segmenter entre commerce alimentaire et non alimentaire est le fait que les décisions antérieures en matière de réglementation du temps de travail se différenciaient selon ce clivage (16). Les comptes rendus de Mademoiselle Deguy, inspectrice du travail en charge de cette branche, permettent de penser que l'existence de leaders capables de mener les délégations dans la négociation est un élément pris en compte. L'arrêté correspondant est signé le 28 mai 1945, mais on assistera encore à quelques tergiversations notamment pour la prise en compte du clivage entre grossistes (et «demi-gros») et détaillants et pour l'inclusion des magasins succursalistes. Non prévues dans l'arrêté primaire, ces distinctions seront prises en compte lors de la publication des classifications en août.

Le canevas sur lequel sont bâtis ces arrêtés primaires est donc relativement homogène. Mais la lecture plus fine des champs d'application d'une part et des définitions de catégories génériques de l'autre permet de penser que les régulations de branche existant avant la guerre ont largement été reprises, y compris dans leur diversité. Qui plus est, la procédure conduit à faire se rencontrer et négocier les acteurs de l'industrie en question, voire même à les constituer. La question qui surgit logiquement, après cette première phase, est donc celle de la prise en compte des résultats de ces négociations dans les décisions ministérielles ultérieures.

(15) L'article 1 de l'arrêté du 28 mai qui définit le champ d'application de la règle donne ainsi de ce champ une définition large («les commerces de gros et de détail de l'alimentation») qui est ensuite restreinte par le rappel des pratiques existantes: «L'arrêté ne s'applique pas aux commerces de l'alimentation visés expressément par les arrêtés fixant les salaires d'une branche d'activité déterminée». L'article 2 donne immédiatement ensuite une définition du champ fondée sur les nomenclatures d'activité, sans pour autant que ces branches d'activité exclues ne soient précisées.

(16) Le clivage a perduré et demeure l'une des clefs pour comprendre les raisons pour lesquelles les hypermarchés, dans les années de leur fort développement (décennies 70 et 80) ont continûment revendiqué leur appartenance au commerce à prédominance alimentaire.
Les « décisions » de classification

Le second temps de la procédure est celui du classement des emplois dans les catégories définies par l'arrêté primaire. Il se termine par une «décision portant classification des emplois» signée du ministre comportant une liste d'emplois classés dans les catégories de l'arrêté correspondant et permettant donc de déterminer les salaires. Cette décision est, du moins pour les dossiers détaillés que nous avons consultés concernant le commerce alimentaire, précédée d'un rapport circonstancié de l'inspecteur du travail qui a supervisé la phase de négociations précédentes (17). Le rapport explicite les points d'accords, propose des solutions de décision pour les points de désaccords et peut même proposer que la décision ne suive pas tous les points d'accord dégagés lors des négociations (18). Il débouche donc sur une proposition faite au ministre. Dans le cas où des classifications existaient dans les conventions collectives d'avant-guerre, elles peuvent servir de base aux discussions, voire être purement et simplement reprises dans la décision: ce fut le cas notamment pour la décision concernant les ouvriers de la métallurgie parisienne datée du 17 juillet(19).

La distinction entre une première phase s'achevant par la publication de l'arrêté primaire et une seconde conduisant à la production de cette décision est une opération logique. L'examen des délais effectifs entre les deux signatures du ministre nous permettra de constater que cette distinction chronologique n'est pas valable sur l'ensemble de la période. On examinera ensuite les techniques de classification utilisée pour repérer le poids des coutumes professionnelles et des précédents dans ces élaborations. Le constat de cette influence remet-il en cause l'idée

(17) Lesquelles négociations se déroulent entre délégations dûment constituées de représentants patronaux et syndicaux, hors la présence de l'inspectrice du travail - ou de quelqu' autre représentant du ministère du Travail - quand elle n'est pas expressément invitée pour résoudre des difficultés de négociation. On peut donc penser que leur fonctionnement ne tombe pas sous le coup de la critique de F. Sellier que nous avons rapportée.

(18) Dans le cas des commerces alimentaires, l'inspectrice du travail constate bien qu'il y a un accord entre syndicats et employeurs pour classer les vendeuses dans des catégories proches de celles de leurs collègues hommes, mais, par souci d'harmonie avec les autres métiers de vendeuses, elle préconise de diminuer l'indice proposé par les représentants de la profession. De même, elle propose au ministre de ne pas suivre l'accord particulier des commerces de charcuterie quant au classement des ouvriers les plus qualifiés.

(19) Dont l'article 1 précise: «Les différents métiers et emplois des industries de la production et de la transformation des métaux de la région parisienne sont classés dans les catégories fixées par l'article 4 de l'arrêté susvisé du 11 avril 1945 conformément au tableau annexé à la présente décision. Ledit tableau reproduit la nomenclature des emplois et métiers de la convention collective de travail du 12 juin 1936 concernant les ouvriers métallurgistes de la région parisienne » (JO 1945, page 4433). 
que l'ensemble de cette réglementation produite par le ministère serait cohérente? On s'interrogera donc sur la qualité de cette couverture réglementaire ainsi produite pendant le ministère Parodi.

\section{À partir de juin, des décisions concomitantes}

La durée de cette seconde phase peut être plus ou moins longue et elle peut être plus ou moins imbriquée dans la première. La première décision de classification publiée est ainsi celle du bâtiment et travaux publics de la région parisienne datée du 14 mai soit moins d'un mois après l'arrêté, alors qu'il faudra attendre le 17 juillet soit trois mois après l'arrêté pour la publication de la décision concernant la métallurgie. Le délai peut être encore plus nettement raccourci et arrêté et décision signés du même jour.

On a pu repérer pour 1945 un corpus de quelque quarante-quatre textes de décision correspondant à une première énonciation de classification suivant les normes énoncées dans l'arrêté correspondant. Parmi ces décisions, vingt-quatre sont datées du même jour ou du lendemain de l'arrêté; sept sont signées dans le mois qui suit, huit dans le trimestre, et quatre dépassent ce délai. À examiner ces délais en fonction des dates de publication, il apparaît clairement un changement dans la politique du ministère: les décisions concomitantes des arrêtés (aucun ou un jour de délai entre les deux signatures) correspondent à des arrêtés signés après le 10 juin, à l'exception de la décision concernant les employés de service et de bureau dont la fonction est interprofessionnelle qui date du 16 mai. À l'inverse, les arrêtés pour lesquels on observe un délai supérieur à un jour entre les deux signatures sont tous signés avant le 19 juin. On peut donc supposer qu'après cette date, les arrêtés non suivis immédiatement de décisions sont considérés comme n'en nécessitant point.

L'examen des documents du dossier concernant les commerces alimentaires conduit à minimiser la portée de ce constat. Les comptes rendus de séance de négociation mettent bien en évidence que la question des salaires effectifs et des classifications, notamment celles des vendeuses, est posée dès le début de la procédure, c'est-à-dire dès avril. En signant les textes le même jour à partir de la mijuin, on peut donc estimer que le ministère reconnaît que cette dynamique de négociation l'emporte sur la logique administrative consistant à séparer les deux phases.

\section{Techniques de classification}

Les canevas d'élaboration de ces textes de décisions sont unifiés. Le texte de la décision proprement dite est fort bref et a pour fonction quasi unique l'introduction d'un tableau de classification établi en utilisant les catégories professionnelles définies dans l'arrêté concerné. Lequel tableau peut être plus ou moins détaillé et plus ou moins fourni : pour l'«industrie du dragage » le texte du tableau n'occupe pas même une colonne soit moins d'un tiers de page du Journal officiel du 22 juillet; pour les industries du verre, la décision du 29 mai n'occupe pas moins de treize pages complètes. Les techniques de description des postes et emplois utilisées dans ces décisions de classification ne sont pas uniformes. Si l'on reprend les catégories utilisées pour analyser les avenants «classifications» des conventions collectives de branche (SAGLIo 1986), on peut classer la plupart des textes comme relevant de la catégorie que nous avions dénommée «Parodi», mais il apparaît également, notamment dans des secteurs fortement structurés par l'action syndicale (industries du verre, industries graphiques) des textes qui reprennent la forme «Parodi amélioré»(20). Parfois les deux techniques de description apparaissent simultanément dans le même texte, mais pour des sous-branches différentes. On peut noter encore que la référence aux diplômes est, dans ces textes de 1945, tout à fait exceptionnelle. On peut donc penser que, sur ces questions de technique de classification, ce sont bien les coutumes antérieures de chaque secteur professionnel qui sont reprises.

L'examen plus détaillé du dossier du commerce alimentaire permet de mieux comprendre les difficultés de cette seconde phase de négociation. On a vu que le premier clivage dans les secteurs du commerce avait consisté à séparer les commerces alimentaires et non-alimentaires. Dans la branche des commerces alimentaires, un second clivage joue lors de cette seconde phase qui distingue les commerces de gros et semi-gros d'une part et les commerces de détail de l'autre. Les maisons à succursales, dont le poids économique n'est pas négligeable, et qui ont l'expérience de négociations sociales notamment parce qu'elles négocient régulièrement avec les syndicats de salariés - et notamment la CGT parisienne-les modalités de rémunération des gérants non salariés, choisissent de se rattacher au commerce de détail. Les rapports de l'inspectrice du travail évoquent également la difficulté de structurer la délégation patronale: le choix de la bonne personne pour mener les négociations aurait fortement joué dans la négociation salariale. Ces préoccupations semblent avoir été dominantes pendant toute la première période de la négociation soit de la fin mars au début juin 1945. Ce n'est qu'une fois ces problèmes à peu près réglés que la négociation sur les catégories de classement des salariés a produit des résultats formalisés soit à partir de début juin 1945. Dès la mi-juillet le canevas de l'accord à proposer à la signature du ministre est prêt. La

(20) Dans la première catégorie, les postes sont décrits par leur simple dénomination, soit en quelques mots; dans la seconde, les fonctions, responsabilités et compétences sont plus nettement définies et chaque description de poste s'avère nettement plus longue. 
décision sera signée le 11 août 1945, le même jour que la décision concernant les commerces de gros de l'alimentation. Pour le commerce de détail, sept sous-branches sont spécifiées et onze pour les commerces de gros. La logique des clivages entre sous-branches dans la même «industrie» transparaît donc à l'occasion des négociations de préparation de cette seconde phase.

\section{Des procédures incomplètes?}

Si toutes les «décisions de classification des emplois » repérées en 1945 correspondent bien à un «arrêté portant fixation des salaires », l'inverse n'est pas vrai et on peut observer un ensemble d'arrêtés orphelins, c'est-à-dire qui ne sont pas suivis d'une ou plusieurs décisions de classification correspondantes.

Une première série correspond à des industries des transports - terrestres, fluviaux, maritimes, ferroviaires - pour lesquels les arrêtés sont le plus souvent interministériels, cosignés avec le ministre des Transports qui, déjà à l'époque, a en charge l'inspection du travail dans ces secteurs. Certains de ces textes pourraient facilement être assimilés aux autres arrêtés de fixation des salaires signés par le ministre du Travail: les techniques de description et de classement des postes se ressemblent, les catégories génériques sont très proches et les échelles hiérarchiques comparables. Parfois cependant, ces arrêtés interministériels relèvent de techniques de classification différentes; mais on peut supposer qu'il s'agit aussi de maintien de traditions professionnelles spécifiques comme dans les mines(21).

Une seconde série de ces arrêtés orphelins correspond à des industries aux emplois peu nombreux ou faiblement structurées et pour lesquelles on peut penser que les catégories produites dans l'arrêté sont suffisantes pour effectuer le classement des emplois(22). Quelques-uns également sont des arrêtés pour lesquels les décisions correspondantes seront effectivement prises, mais en intégrant les industries en question dans un ensemble plus vaste: les ouvriers de la fourrure seront ainsi classés dans les industries du vêtement, les ouvriers du commerce des combustibles dans les commerces non alimentaires.

(21) L'arrêté «Salaires dans les mines» du $1^{\text {er }}$ juin, ne fait même pas référence aux catégories de salaires et aux échelles hiérarchiques. Par contre, il détaille assez précisément le calcul des salaires horaires. Ce régime est étendu aux mines de fer et de potasse le 19 juillet, aux mines de métalloïdes et de métaux non ferreux le 22 août. Des arrêtés proches sont pris ce même 22 août pour les exploitations de bauxite et pour celles d'ardoises. Tous ces arrêtés sont cosignés avec le ministre de la Production industrielle.

(22) C'est le cas des «industries» suivantes: scaphandriers de mer, scaphandriers de rivière, ouvriers boulangers faisant la panification, ouvriers de l'orfèvrerie, personnel des crèches...
Une troisième série d'arrêtés orphelins mérite une mention particulière : les neufs arrêtés concernant les techniciens, dessinateurs, ingénieurs et cadres dans lesquels ces catégories ne sont pas associées à celle des employés ne sont en effet pas suivis de décisions correspondantes. Pour une part, c'est le fait que ces arrêtés sont tardifs - quatre sont pris au cours de la seconde quinzaine du mois de décembre. Mais c'est aussi le fait que la technique utilisée pour ces arrêtés ne nécessite pas la production d'une décision de classification des emplois pour les rendre opérationnels. Il suffit d'appliquer aux emplois considérés les critères d'évaluation énoncés dans l'arrêté fixant les appointements. Les longs arrêtés décrivant les emplois de techniciens, dessinateurs et agents de maîtrise du 25 septembre pour le bâtiment et les travaux publics ou le $1^{\mathrm{er}}$ novembre pour la chimie peuvent ainsi être considérés comme se suffisant à eux-mêmes pour être appliqués.

\section{Quelle couverture régulatoire?}

Le respect de la procédure complète de «remise en ordre des salaires» aurait suscité la production d'un nombre de textes ministériels difficile à évaluer mais en tout état de cause fort important. $\mathrm{Si}$ l'on suit en effet les exemples des industries les plus structurées, il conviendrait de disposer, pour chaque «industrie» de trois, voire quatre arrêtés différents selon les regroupements de catégories opérés (ouvriers/employés/techniciens, agents de maîtrise, dessinateurs/ingénieurs et cadres) et autant de décisions, sans même compter les textes concernant les métiers «interprofessionnels».

Au moment du changement de ministre (23), on constate que les industries pour lesquelles une telle réglementation complète est en place sont rares. Pour cette analyse, on a relevé systématiquement tous les textes, arrêtés et décisions, ayant pour objet les rémunérations et n'étant ni interprofessionnels, ni «géographiques»(24). On obtient ainsi une liste de cinquante-sept «industries» pour lesquelles il existe au moins un arrêté (25). L'examen rapide des dénominations conduit à supposer que la définition des «industries» a été une affaire plus pragmatique que systématique. À côté de branches déjà connues avant guerre pour la négociation collective (métallurgie, chimie, banques, industries graphiques, etc.), on voit apparaître des secteurs très restreints (scaphandriers de rivière, fabrication de vêtements

(23) Le 21 novembre 1945, Ambroise Croizat succède à Alexandre Parodi, démissionnaire le 21 octobre.

(24) Par ce terme on entend les arrêtés délimitant les zones en matière de niveau de salaires ou les arrêtés d'extension rendant opératoire un texte dans une zone non prévue initialement.

(25) On a regroupé en une seule «industrie» les différents secteurs des mines autres que les «mines de combustibles minéraux solides et schistes bitumineux » dans la mesure où les arrêtés en question sortent le même jour et sont identiques, au pourcentage de décalage autorisé par rapport aux salaires de la métallurgie près. 
imperméables en caoutchouc...) ou des secteurs nouveaux aux contours mal définis (commerces non alimentaires, commerces alimentaires...). Au total cependant, on peut estimer que la couverture des arrêtés de salaires pour les ouvriers est à peu près réalisée sur l'année 1945(26), et donc avant même que ne se manifestent les effets de la nomination d'Ambroise Croizat comme ministre.

Sur ce total, seules trois «industries» («industries métallurgiques», «industries du bâtiment et des travaux publics», «industries chimiques et parachimiques») disposent, à la fin 1945, de trois arrêtés. On peut, à ce total, ajouter trois autres branches pour lesquelles le périmètre de l'arrêté initial est plus large que celui des décisions, mais dont on peut penser qu'elles disposent effectivement de textes de classification couvrant la quasi-totalité de leurs salariés: les assurances dans le secteur «banques, Bourse et assurances », le notariat dans le secteur des «professions libérales» et certains secteurs du commerce de gros non alimentaire, bien que n'ayant que deux textes d'arrêtés, voient cependant l'ensemble de leurs personnels salariés couverts par cette procédure. Ce sont donc six «branches» pour lesquelles l'ensemble des arrêtés nécessaire à la réglementation des salaires est produit à la fin 1945(27). Quatre autres branches ont deux textes d'arrêtés publiés, mais ne disposent pas d'arrêtés permettant le classement de leurs cadres voire de leurs techniciens et agents de maîtrise.

Quant aux décisions de classification, on peut constater que vingt-deux de ces cinquante-sept «industries» dont l'identité est précisée par un des arrêtés (soit 39\%) ne disposent en fin de période de la décision de classification correspondante. Compte tenu de l'observation que nous avons faite précédemment sur les délais de signature des décisions, on est donc amené à penser que, pour la plupart de ces branches, il n'y a plus guère, à la fin de l'automne 1945, de décisions à attendre pour les ouvriers et les employés. Par contre, pour ce qui concerne les techniciens, les agents de maîtrise, les ingénieurs et les cadres, les textes commencent juste à sortir. Mais déjà, l'analyse plus fine que nous venons de mener conduit à une autre indication; dès ce moment, il n'y a pas un modèle unique de régula-

(26) Nous n'avons pas trouvé de documents faisant cette analyse systématique à partir de la nomenclature des industries et professions de la statistique générale de la France utilisée à l'époque. En recoupant avec une liste des conventions collectives de branches de niveau national existant aujourd'hui, on peut détecter quelques «trous» de la réglementation à la fin 1945. Une première série renvoie à des textes en cours d'élaboration (caoutchouc, réparation automobile qui «sortiront » début 1946). D'autres, notamment dans les services sociaux n'existent pas encore en tant que tels. Cette évaluation sommaire nous conduit cependant à penser que la couverture réalisée peut être considérée comme quasi-complète.

(27) On rappelle que ces arrêtés sont censés prendre effet au 15 mars 1945. tion de branche, dans lequel la procédure complète nécessiterait trois ou quatre arrêtés et autant de décisions, mais bien une variété de modèles. Lorsqu'on fait le bilan à la fin du ministère Parodi, tout se passe donc comme si les logiques et les coutumes régulatoires propres à chaque branche étaient de nouveau prises en compte.

\section{Reprises et révisions}

Il serait toutefois erroné de considérer que tout ce processus de production de la réglementation en matière salariale se déroule de manière fluide et cohérente. L'encre à peine sèche, les textes sont remis en cause et révisés. Et les acteurs de terrain s'entendent pour coordonner leurs critiques. La précision des catégories et des classifications n'est alors que le masque de l'inefficacité de la régulation.

\section{Remises en cause}

Très rapidement, on voit en effet publier des arrêtés modificatifs qui transforment sur des points parfois substantiels la réglementation première. Pour la métallurgie, la première de ces modifications intervient moins de quinze jours après la publication de l'arrêté du 11 avril; elle concerne notamment le point délicat de la rémunération des ouvriers aux pièces: le taux horaire maximal pris pour base de calcul des tarifs de pièces est porté de 120 à $125 \%$ $\mathrm{du}$ taux horaire minimum. Soit une augmentation des salaires de $4 \%$ pour la plupart des ouvriers. Après la publication de l'arrêté du 31 mai, on peut estimer que cette réglementation n'a plus qu'un caractère vaguement indicatif. C'est bien l'indice que la négociation professionnelle reste active et que les acteurs se sont mis d'accord pour critiquer la règle étatique dès son énonciation.

Les difficultés de la régulation de type interprofessionnelle ont également surgi rapidement. On observe ce fait pour la tentative qui concerne le plus grand nombre tout à la fois de salariés et d'entreprises qui est celui de la régulation des conditions de salaires pour les «employés de bureau et services annexes dont la fonction présente un caractère interprofessionnel». L'arrêté définissant les professions concernées et la décision de classification correspondante sont tous deux datés du 16 mai et publiés au Journal officiel le 19. Ils sont immédiatement pris en compte dans certaines branches: l'arrêté concernant les « salaires des commerces non alimentaires», pris le 17 mai, celui des «salaires des employés des banques, de la Bourse et des assurances», pris le 18 mai sont également publiés le 19 et y font référence.

Un arrêté et une décision de classification, signés le 28 mai et publiés le 31, apportent déjà des modifications sensibles aux textes du 16 mai : les coefficients de certaines catégories sont modifiés - et 
en pratique relevés de 3 ou 5 points sauf pour la catégorie de base et les deux catégories supérieures - l'intégration du treizième mois dans la rémunération prise en compte n'est plus mentionnée explicitement, les marges de variation entre salaire minimum et maximum par catégorie sont revues à la hausse, enfin les classifications sont revues à la hausse pour certains emplois. Ce nouveau texte est applicable au $1^{\mathrm{er}}$ mai. Ce qui entraîne en cascade deux arrêtés apportant les révisions similaires pour les «banques, Bourse et assurances» d'un côté et pour les «commerces non alimentaires » de l'autre. Simultanément, c'est-à-dire avec le même jour de signature de l'arrêté et la publication dans le même numéro du Journal officiel, un arrêté prévoit que les branches de la métallurgie, du BTP et de la chimie pourront déroger à cette réglementation. Cette dérogation autorisée peut sembler de pure forme puisque l'article 2 de l'arrêté stipule expressément que le salaire réel devra néanmoins être compris dans la fourchette définie par l'arrêté auquel il est autorisé de déroger(28). Dès le 4 juin, les augmentations de salaires pour les employés des trois branches concernées sont alignées sur les augmentations prévues pour les ingénieurs techniciens agents de maîtrise et cadres et donc privilégient les logiques «industrielles » consistant à regrouper les employés techniciens et agents de maîtrise dans les mêmes régulations plutôt que la logique interprofessionnelle qu'avait tenté de mettre en place le ministère.

Le 12 juin, un nouvel arrêté concernantl'ensemble de ces employés de bureau et services annexes modifie de nouveau la grille des coefficients - en créant un nouvel échelon dans la première catégorie des employés - et exclut explicitement le treizième mois du calcul de la rémunération régulée si celui-ci était prévu antérieurement par des conventions. Le

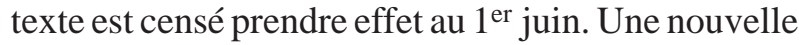
fois, la classification des emplois correspondante est revue et complétée, et des arrêtés modificatifs sont pris pour les banques, Bourse et assurances et pour les commerces non alimentaires.

En moins d'un mois, le ministre a donc signé trois versions différentes de l'arrêté et autant de décisions de classification. Une semblable valse hésitation manifeste à l'évidence les réticences auxquelles se heurte le projet d'une telle régulation.

(28) Article1: «Par dérogation aux dispositions de l'arrêté du 16 mai 1945 précité, les salaires des employés de bureau et services annexes visés à l'article $1^{\text {er }}$ dudit texte seront fixés par arrêté spécial dans les industries des métaux, les industries chimiques, les industries du bâtiment. Article 2. - Les arrêtés spéciaux prévus à l'article précédent pourront prévoir, pour les travailleurs définis ci-dessus, une classification différente de celle établie par l'arrêté précité du 16 mai 1945, sans toutefois que le salaire réel d'un salarié puisse être inférieur au minimum ou supérieur au maximum fixé pour sa catégorie d'emploi par ledit arrêté du 16 mai 1945.»
On peut se demander si un projet similaire de régulation interprofessionnelle a existé pour les appointements des ingénieurs, cadres techniciens et agents de maîtrise. Signé le 4 juin, l'arrêté d' «ajustement provisoire des appointements des ingénieurs, techniciens, agents de maîtrise et agents des cadres supérieurs de toutes professions » comprend bien cette référence interprofessionnelle dans son intitulé. Par contre, le texte fait une référence explicite à la régulation des rémunérations instituée par les conventions collectives postérieures à 1936 quand elles comportaient mention explicite de ces catégories professionnelles, ce qui produit une certaine complexité du texte ( $c f$. encadré 4$)$. Les quelques textes concernant les cadres et ingénieurs publiés fin 1945 amènent à constater que la différenciation selon les branches est forte. Les modes de description et de classement des postes sont proches pour les branches «industrielles » et notamment pour la métallurgie, le BTP et la chimie. Par contre, celui mis en œuvre dans le texte des personnels d'encadrement des auxiliaires de justice et du notariat du 20 décembre, et surtout dans celui des «cadres de commandement et cadres techniques» des commerces de gros et demi-gros non alimentaires daté du 27 décembre utilisent une tout autre technique de description des postes et de justification des hiérarchies salariales.

L'examen de ces diverses versions des textes à vocation interprofessionnelle et le fait que les branches industrielles où la négociation est la plus structurée obtiennent la reconnaissance de leur autonomie en matière de régulation manifestent à l'évidence les obstacles auxquels s'est heurtée la tentative du ministère.

\section{Des divergences sur les fondements des différenciations salariales?}

On peut esquisser une première ligne d'explication de ces réticences des autres acteurs à accepter ce mode de régulation salariale qui serait liée à la justification même des hiérarchies salariales. La technique de classement hiérarchique utilisée par le ministère est en effet fondée sur le positionnement des individus dans un système unifié et homogène de classement des professions et des métiers: il existerait ainsi une hiérarchie professionnelle ou un ordre salarial unifiés(29). On revient ainsi à une rémunération fondée sur le rang, dont on sait

(29) Anne-Sophie Beau (2001) utilise un raisonnement identique pour expliquer l'existence de l'arrêté concernant les emplois de bureau à fonction interprofessionnelle: «Le ministre du Travail prend acte, ensuite, de l'inadéquation entre le cadre de la branche, choisi pour la définition des hiérarchies professionnelles, et celui des postes de travail qui, comme les emplois de bureaux, sont présents dans tous les secteurs d'activité.» La détermination du cadre et du niveau de la régulation salariale serait donc ainsi réduite à n'être qu'une affaire fonctionnelle dont il suffirait de «prendre acte». 
Encadré 4

Arrêté du 4 juin 1945: «ajustement
provisoire des appointements des
ingénieurs, techniciens, agents
de maîtrise et agents des cadres
supérieurs de toutes professions "

«Article 1. - Les appointements des ingénieurs, techniciens, agents de maitrise et agents des cadres supérieurs de toutes les professions seront provisoirement calculés à raison de $166 \%$ de la valeur réelle qu'ils avaient au mois de juillet 1944 .

Cette majoration ne jouera que pour moitié en ce qui concerne la tranche d'appointement qui, en juillet 1944 était comprise entre 15000 et 25000 francs. Elle cessera de s'appliquer pour la tranche audelà de 25000 francs.

Article 2. - Les appointements nouveaux ainsi calculés ne pourront être ni inférieurs à la valeur qu'ils avaient en 1936 multipliés par le coefficient 3,33, ni supérieurs aux mêmes appointements multipliés par le coefficient 3,82 .

Les appointements de 1936 qui devront être pris pour terme de comparaison sont ceux des conventions collectives d'ingénieurs techniciens, agents de maîtrise et agents des cadres supérieurs conclues par application de la loi du 24 juin 1936, dans tous les cas où ces conventions collectives existent. Là où elles n'existent pas, les coefficients ci-dessus seront appliqués aux appointements qui étaient réellement pratiqués à la date du 31 décembre 1936.

Pour les établissements créés postérieurement à cette date, le relèvement de salaire sera établi par comparaison avec les salaires couramment pratiqués en application des règles ci-dessus pour les établissements de la même profession qui existaient à la date du 31 décembre 1936.

Les difficultés qui pourraient surgir de l'application du présent article seront réglées par l'inspecteur divisionnaire du travail et de la main-d'œuvre.

Article 3. - Des dérogations pourront être accordées aux règles qui précèdent à la demande des chefs d'établissement ou des organisations syndicales patronales et ouvrières, lorsque l'application de ces règles ne maintiendrait pas un écart suffisant entre les appointements des ingénieurs techniciens et agents de maîtrise et les salaires des ouvriers ou employés des catégories supérieures placés sous leurs ordres.

Lesdites dérogations seront accordées par le ministre du Travail et de la Sécurité sociale lorsqu'elles auront un caractère national et par l'inspecteur divisionnaire, directeur régional du travail et de la main-d'œuvre, lorsqu'elles auront un caractère régional ou local.

Article 4. - Les appointements des employés de bureau des industries des métaux, des industries chimiques et des industries du bâtiment et des travaux publics seront réajustés provisoirement dans les mêmes conditions.

Article 5. - Les dispositions qui précèdent prendront effet du 15 mars 1945. Elles ne pourront entraîner aucune réduction des appointements régulièrement payés à cette date. » qu'elle est au fondement de la rémunération dans la fonction publique dès l'entre-deux-guerres (30). C'est d'ailleurs cette conception qui sera peu après au fondement de la mise en place des catégories socioprofessionnelles (31).

À l'évidence, et dès le mois de mai 1945, la mise en place d'un tel système se heurte à des réticences et des résistances. Pour les comprendre, il faut revenir au fait que le primat du niveau de la branche dans la régulation salariale légitime des différences de salaires entre des salariés qui relèvent de la même catégorie professionnelle. Un ouvrier hautement qualifié a ainsi un salaire horaire de base plus élevé dans l'industrie du verre que dans les industries textiles. Accepter cette possibilité amène à changer radicalement la justification des salaires (32). Dans la conception où prévaut la rémunération selon le rang, la hiérarchie des salaires fait partie de l'environnement économique des acteurs et le résultat de l'entreprise est le produit de la combinaison des facteurs de production - dont le travail - à des coûts fixés de l'extérieur. Dans la conception où prévaut la négociation, celle-ci est le moyen de répartir la richesse produite: la rémunération du salarié peut donc varier selon la plus ou moins grande capacité de l'entreprise à dégager des marges bénéficiaires, de même qu'elle varie selon les rapports de forces internes entre les différents groupes. On voit bien que c'est là où les syndicats ouvriers sont les plus forts que les rémunérations ouvrières sont les plus élevées. On comprend donc ainsi que la proposition de «mise en ordre» fondée sur des catégories interprofessionnelles ait suscité des réticences du côté des acteurs, notamment syndicaux. On perçoit enfin que, au-delà des récriminations sur les règles de fond, c'est la conception même des institutions intermédiaires (entreprises et branches) et de leur place dans la régulation salariale qui est en cause.

Qui plus est, la mise en place de cette régulation centralisée se heurte également à des obstacles plus strictement techniques. En matière de niveau des salaires, la régulation se complexifie au cours de l'année. L'objectif d'effectuer la «remise en ordre» semble passer par l'énonciation d'un corpus de textes réglementaires ayant validité au même moment et couvrant un maximum de situations d'emplois différentes. Les différents arrêtés, quelle que soit leur date de signature, sont censés prendre effet au 15 mars 1945 et se caler par rapport à la référence que constitue le salaire horaire de l'ouvrier de base

(30) On peut se rapporter notamment aux justifications qu'en donne L. Duguit (1923) dans ses travaux.

(31) Cf. Notamment Desrosières et THÉVenot (1988) qui supposent bien que la nomenclature qui se met en place à l'époque est «unidimensionnelle» et, précisent-ils, définit des niveaux «hiérarchisés selon des durées et des types de formation» $(16)$

(32) On peut suivre ici les raisonnements de R. BIERNACKI (1995) sur les différents modes de formation des salaires. 
de la métallurgie parisienne. Dans la pratique les arrêtés ne définissent pas strictement un niveau de salaires, mais une plage. Qui plus est, la borne supérieure de cette plage est définie, depuis août 1944, non comme un salaire individuel maximum mais comme un salaire moyen maximum.

En examinant la succession des textes publiés en 1945, on peut en déduire en creux différentes tactiques mises en œuvre pour contourner cette réglementation. En premier lieu, on cherche à décaler la borne supérieure en élargissant la plage: un arrêté du 31 mai sur la détermination des salaires maxima stipule que l'ampleur de celle-ci est multipliée par deux: «La différence entre les taux de salaires minima et maxima tels qu'ils résultent dans chaque catégorie professionnelle, des arrêtés énumérés ciaprès est multipliée par le coefficient 2 pour les travailleurs rémunérés à l'heure: [...]». Et suit la liste exhaustive des arrêtés déjà publiés concernant les ouvriers. En second lieu, comme on l'a vu pour les employés, on peut modifier la base de calcul du salaire de référence, notamment en n'y intégrant plus le treizième mois.

La troisième tactique consiste à exclure progressivement de la réglementation certaines formes de rémunération, notamment pour les ouvriers payés au rendement d'une part et les vendeurs rémunérés au pourcentage ou à la guelte de l'autre. L'évolution est nette pour les textes de la métallurgie puisqu'à partir du 31 mai, on peut considérer la réglementation concernant les ouvriers payés au rendement comme non contraignante.

\section{La double opposition \\ des acteurs de terrain}

Il n'est certainement pas difficile de comprendre les raisons et les processus qui conduisent à ces tergiversations ministérielles. L'ampleur et la diversité des problèmes à résoudre, les difficultés de la conjoncture économique, celles de la coordination gouvernementale avec le ministère des Finances sont autant de raisons parfaitement suffisantes pour comprendre que la politique menée en matière salariale ne soit pas totalement cohérente et fixée sur l'ensemble de la période.

Il est plus important ici de se demander ce que ces flottements et corrections successives ont produit sur les comportements et les stratégies des autres acteurs. L'examen du dossier du secteur des commerces alimentaires permet de tracer quelques hypothèses en la matière. On l'a vu précédemment, la période du printemps est celle de l'amorce des négociations et des difficultés à constituer et à donner de la cohérence à la délégation patronale. Les négociations proprement dites sur les classifications et les salaires, pour l'ensemble et pour les différentes sous-branches se déroulent pour l'essentiel au cours des réunions de juin. Les indications présentes dans le dossier laissent supposer que, dans cette première phase, syndicats et patronat partagent une préoccupation commune de faire patienter leurs mandants le temps de faire aboutir les négociations. Aucun indice ne semble en effet signaler des mobilisations particulières d'un côté ou de l'autre.

La situation change à l'été, et plus nettement à partir de la fin juillet. À la mi-juillet en effet, les négociations sont considérées comme terminées, les résultats sont là; l'inspectrice du travail en charge du dossier rédige son rapport et le remet au ministre. Bien souvent, ces négociations ont abouti à des accords en bonne et due forme, signés des employeurs et des syndicats de salariés et transmis au ministère. Les courriers conservés amènent à penser que durant cette période les employeurs et les salariés conjuguent leurs pressions sur le ministère pour tenter d'accélérer le processus de signature et de publication. Cette pression atteint son acmé en août (voire début septembre pour les commerces de gros) lorsque les acteurs savent que les documents sont prêts et n'attendent plus que la signature ministérielle. On peut alors assister à des numéros de surenchère. Ainsi le secrétaire de la fédération CGT de l'alimentation proteste vigoureusement le 20 septembre auprès du ministre à propos de la situation des entrepôts (où l'on attend encore la publication de la décision correspondante): «C'est là une situation qui, si elle devait durer encore longtemps, ne manquerait pas d'amener de la part de nos corporants une réaction plus ou moins violente. Jusqu'à maintenant nous les avons engagés à la patience mais nous ne saurions conserver cette attitude indéfiniment au risque de voir les ouvriers se retourner contre nous-mêmes». Et il termine sa lettre: «Nous craignons fort que nos conseils ne soient bientôt plus suivis, si vos services avec ceux de l'économie nationale, ne trouvent pas une solution».

À l'occasion, le patronat relaye une pression du même type. Le secrétaire général du patronat de l'épicerie écrit ainsi au ministère le 13 août: «Nous avons l'honneur de vous signaler que nos adhérents nous indiquent une effervescence croissante dans le personnel employé et ouvrier. Il y a de nombreuses menaces de grève, le mécontentement est général». Il dénonce alors la lenteur de l'administration: «Le personnel n'admettra jamais, en effet, que des textes en préparation depuis plusieurs mois ne soient pas encore sortis. Pour notre part, nous ne voulons pas que l'on nous impute à faute ce qui n'est que retard administratif ».

La troisième phase s'ouvre avec la publication des textes de décisions. La procédure employée, en donnant à la règle la forme d'une décision ministérielle, déresponsabilise les acteurs de terrain visà-vis de la décision. Elle pousse à l'évidence les syndicats ouvriers à soutenir les revendications catégorielles, voire à faire de la surenchère. Et ce d'autant plus que l'expérience des mois d'avril sur le texte des ouvriers de la métallurgie et de mai sur 
Tableau 2

Nombre des appellations d'emploi dans les décisions de la métallurgie

\begin{tabular}{|c|c|c|c|c|c|c|c|}
\hline Dates & MO & MF & OS & Assimilés prof. & P1 & P2 & P3 \\
\hline $17 / 7 / 1945$ & & & 239 & 50 & 101 & 30 & 17 \\
\hline $6 / 10 / 1945$ & 12 & 61 & 530 & 43 & 161 & 55 & 28 \\
\hline
\end{tabular}

les employés dont la fonction est interprofessionnelle leur a enseigné que la position du ministère n'était pas totalement rigide. La conjoncture reste revendicative pour bien des salariés. On sait que l'inflation est considérable et que le pouvoir d'achat des salaires réels s'affiche le plus souvent en baisse nette. Et les difficultés d'approvisionnement ou de logement ne sont pas des éléments qui font diminuer l'insatisfaction des salariés. Les syndicats vont donc soutenir les revendications de reclassement ou de correction des classifications. Ainsi le syndicat CGT des établissements Casino de Beaucaire va-t-il se mobiliser dès septembre pour obtenir une revalorisation de la catégorie des «remplisseurs de fûts». Le conflit va durer près d'un an pour obtenir que la revalorisation soit rétroactive au 15 mars.

Mais les organisations patronales ne sont pas en reste quand il s'agit de critiquer des décisions qui ne sont pas totalement conformes à leurs souhaits. Ainsi, dès le 23 août, le patronat de la crémerie fait parvenir une lettre critiquant la décision du 11. En octobre une note non signée rappelle la préférence patronale pour la rémunération au pourcentage des vendeuses de laiterie et pour une revalorisation de leur coefficient.

\section{L'explosion des catégories et des sous-branches}

La conjonction de la relative faiblesse du ministère et de la double opposition des acteurs produit un affaiblissement de la régulation. L'une des manifestations les plus évidentes est l'inflation du nombre et de la taille des textes réglementaires. On a vu le processus d'inflation du nombre de textes en suivant la valse-hésitation sur la question des employés dont la fonction est interprofessionnelle. Les courriers de protestation, notamment des employeurs, sont remplis de réclamations sur le sujet lorsqu'ils demandent, avec plus ou moins d'humour, de vérifier si la liste des textes qu'ils doivent prendre en compte pour fixer les salaires est bien complète (33).

(33) Le dossier 19760121 article 209 conserve une correspondance plus tardive (avril 1949) où, en réponse à une demande du «Bulletin du commerce et de l'alimentation », le ministère donne la liste des textes à prendre en compte pour fixer la rémunération des personnels. Elle comporte trente-cinq références pour les commerces alimentaires et vingt-sept pour les commerces non alimentaires et, significativement, ne fait pas pour autant mention des arrêtés du 16 mai et de leur suite concernant les employés dont la fonction est interprofessionnelle.
Mais l'inflation ne concerne pas que le nombre de textes applicables. La taille des textes de classification et leur complexité s'accroissent fortement. Sur la durée, dans l'année 1945, on peut observer le processus qui conduit à une multiplication du nombre des sous-branches répertoriées et du nombre des appellations d'emploi citées. L'exemple des ouvriers de la métallurgie est fort significatif de ce processus qui affecte également la plupart des branches. Pour les seuls ouvriers de la métallurgie, et en ne prenant pas en compte les textes qui portent sur la question des zones de salaires, ce sont cinq textes qui scandent la production de la régulation en 1945 soit:

- l'arrêté du 11 avril, modifié par arrêté du 24 avril définissant les catégories génériques et les niveaux de salaires;

- les décisions du 17 juillet, 6 octobre et 20 décembre portant classification des emplois.

Le premier arrêté ne comporte pas de définitions de sous-branches correspondant à des secteurs industriels de la métallurgie. La décision du 17 juillet fait mention d'une catégorie générale et de cinquante-quatre sous branches, celle du 6 octobre passe à soixante et une sous-branches, et celle du 20 décembre n'est que partielle. Le tableau 2 présente, par catégorie professionnelle, l'accroissement du nombre d'appellations d'emploi présentes dans chaque texte.

Ce processus d'inflation du nombre de catégories peut également s'observer, quoique de manière moins nette pour ce qui concerne les employés. Pour les autres groupes professionnels, la période retenue (l'année 1945) n'est pas suffisante pour évaluer le processus. On peut toutefois remarquer que, pour les ingénieurs et cadres et en retenant comme période d'observation celle qui va jusqu' aux grèves de mai 1947 et au départ des communistes du gouvernement, la situation est différente dans la mesure où l'arrêté de définition des catégories pour les appointements n'est pas suivi de la publication d'une décision de classification correspondante. Quant aux classifications des ETDA publiées en 1946 sous la signature d'Ambroise Croizat, elles le seront sur la base de textes régionaux, voire de champs d'application nettement plus limités.

Dans d'autres branches, le même processus peut être identifié quand on constate l'ampleur des textes de classification. Ceux des industries textiles ou du vêtement sont ainsi particulièrement fournis. Dans le cas de la bonneterie, on peut même observer la précision apparente de la régulation qui utilise des 
échelons intermédiaires avec des variations de coefficient point par point.

Cette inflation du nombre des appellations d'emploi correspond bien évidemment à une accumulation de petits conflits de classement. Le dossier des commerces alimentaires comprend ainsi une série de récriminations, soutenues par les syndicats, mais parfois aussi par les organisations patronales concernant le classement de telle ou telle catégorie locale de salariés. On peut alors penser que, plutôt que d'en appeler à une règle générale qui permettrait de trancher le différend ou au moins d'organiser la controverse, la forme de la régulation adoptée, et cautionnée par le ministère, consiste à ajouter des catégories et des précisions, dans une fuite en avant indéfinie de la production réglementaire. Et les acteurs syndicaux et patronaux semblent s'être adaptés à ce glissement d'une conception conventionnelle vers une conception administrative de la régulation. Il est probable cependant que cette inflation réglementaire ne correspond pas pour autant à une amélioration de l'effectivité de la régulation en matière de régulation salariale.

\section{Une nouvelle définition de la place de l'État}

On est souvent tenté de voir dans cette période de la Libération un moment où une nouvelle régulation se met en place progressivement de manière plutôt maîtrisée par un gouvernement doté d'une forte légitimité. Dans le domaine qui nous intéresse, ce schéma se traduirait par une production régulière de textes venant progressivement compléter et parfaire une régulation des salaires organisée depuis le centre qu'est le ministère du Travail, lui-même travaillant en coordination avec le ministère de l'Économie et des Finances.

\section{Le contrôle des finances}

Déjà sur ce dernier aspect, on sait que la réalité fut nettement moins consensuelle et que les conflits entre les deux ministères furent fréquents. Le ministère en charge des Finances intervient sur de nombreux sujets: ainsi, dans le commerce alimentaire, c'est lui qui bloque la mesure de suppression des heures d'équivalence que demandent les syndicats de salariés, que soutiennent les inspecteurs du travail chargés d'instruire le dossier et que ne défendent qu' assez mollement les patrons ; mais le ministère des Finances y voit un risque de hausses des prix dans le commerce. La politique du ministère des Finances de l'époque est en effet nettement plus influencée par le souci de juguler l'inflation et donc de ne pas laisser glisser les salaires. C'est probablement au fait que cette préoccupation a prévalu le plus souvent que l'on doit l'irréalisme concret des mesures à partir du milieu de l'année 1945 : dans un moment où la hausse des prix se fait sur un rythme annuel proche de $40 \%$, le maintien dans tous les textes signés d'une même référence de base, à savoir le salaire horaire de 20 francs de l'ouvrier manœuvre de la métallurgie parisienne produit un décalage progressif et rapide des règles énoncées par rapport aux réalités. On peut voir dans cette politique de l'époque l'une des origines du décrochage habituel entre les salaires conventionnels négociés et les salaires réels. Même une fois la contrainte de la politique étatique levée, l'habitude de ce décrochage demeurera. Dans l'immédiat, l'effet final sur le niveau moyen des salaires est bien conforme à l'objectif de lutte contre l'inflation: l'évolution des salaires ouvriers a décroché nettement par rapport à l'inflation en 1946 et 1947 (34).

\section{Pousser les autres acteurs à la récrimination}

On attribue le plus souvent à ce phénomène de perte du pouvoir d'achat des salaires, ainsi qu'à des considérations plus directement politiques et notamment à ses liens avec le Parti communiste, la position de défiance par rapport au gouvernement que prend progressivement la CGT. Dans son ensemble, elle n'aurait guère été critique de la centralisation des décisions notamment en matière salariale (35).

L'examen de la production des textes de régulation des salaires et l'examen plus détaillé du dossier de la politique salariale dans les secteurs du commerce alimentaire amènent à produire sur ce point une hypothèse complémentaire. On constate en effet que les syndicats CGT concernés se montrent nettement coopératifs dans toute la phase de préparation des décisions, au point que nombre des textes transmis au ministère jusqu'à juillet 1945 sont des accords collectifs en bonne et due forme.

Les comportements se modifient à partir de la publication des arrêtés et décisions ministériels. Pour les syndicats de salariés comme pour les organisations patronales, le fait que le texte énonçant la régulation paraisse sous la seule signature ministérielle produit le même effet de désengagement

(34) Dans son imposant travail sur les hauts revenus en France, Thomas Piketty établit des séries statistiques cohérentes sur le $\mathrm{XX}^{\mathrm{e}}$ siècle. Pour les années qui nous intéressent ici il retient pour l'inflation et l'évolution du salaire ouvrier moyen les données suivantes: (PIKETTY, 2001, cf. p. 690 col. 5 et p. 679 col.6).

\begin{tabular}{llllll}
\hline & $\mathbf{1 9 4 4}$ & $\mathbf{1 9 4 5}$ & $\mathbf{1 9 4 6}$ & $\mathbf{1 9 4 7}$ & $\mathbf{1 9 4 8}$ \\
\hline Taux d'inflation & $22,30 \%$ & $48,20 \%$ & $52,60 \%$ & $49,40 \%$ & $58,50 \%$ \\
\hline $\begin{array}{l}\text { Salaire ouvrier } \\
\text { moyen annuel } \\
\text { (net nominal) }\end{array}$ & 28531 & 49771 & 70651 & 96900 & 155900 \\
\hline $\begin{array}{l}\text { Croissance du } \\
\text { salaire ouvrier } \\
\text { moyen }\end{array}$ & & $+74 \%$ & $+42 \%$ & $+37 \%$ & $61 \%$ \\
\hline
\end{tabular}

(35) C'est la position reprise encore dans les travaux d'Adam Steinhouse, $c f$. notamment Steinhouse, 2001, pp. 142-147. 
critique: ces organisations n'ont pas grand-chose à gagner à soutenir le texte auprès de leurs mandants et sont plutôt amenées à se solidariser des groupes critiques. En perdant la légitimité qu'avaient les textes de compromis sur lesquels se fondent les règles de fond issues de la négociation, les divers arrêtés et décisions ministériels deviennent en fait des textes fragiles et, dès la fin mai 1945 et les tergiversations sur les textes concernant les employés, on peut penser que les acteurs de terrain savent d'expérience qu'ils peuvent être bousculés.

On peut penser également que les acteurs de terrain apprennent très vite comment contourner les règles étatiques centralisées. L'ampleur du glissement du salaire ouvrier moyen mise en relation avec la fixité de la norme de référence des différents textes officiels en est un indice évident. L'hypothèse est d'autant plus plausible que c'est ainsi l'acteur le moins présent sur le terrain qui prétend imposer les règles pour les relations quotidiennes des deux autres protagonistes. Mais ces accords « de base» ne peuvent être que quasi-clandestins puisqu'ils consistent à contourner la réglementation. La politique gouvernementale de refus de reconnaître la légitimité des compromis contribue ainsi largement au fait que le bénéfice éventuel d'une position coopératrice est difficile à escompter pour le syndicat. La politique salariale du gouvernement de la libération contribue ainsi fortement à renforcer l'une des caractéristiques notables du système français des relations professionnelles qu'est la réticence des acteurs à contracter.

En examinant ainsi dans le détail la politique salariale à la Libération, on est amené à remettre en cause l'idée courante que le rôle hypertrophié de l'État serait dû aux comportements peu coopératifs des autres partenaires du système de relations professionnelles. En disqualifiant les compromis, y compris quand ceux-ci existent effectivement, c'est plutôt l'État qui pousse les autres acteurs à adopter ces comportements dont tout le système pâtira par la suite. On voit sur cet exemple que c'est bien la politique menée par l'État qui contribue alors à renforcer cette propension souvent dénoncée des acteurs à adopter des comportements qui font privilégier la décision étatique à la solution négociée. On peut considérer que la période est en quelque sorte un moment instituant de cette caractéristique.

\section{Imposer un ordre salarial unifié}

Les politiques salariales sont au centre des différends que nous avons ici examinés. Dans ce domaine, la solution imposée par le ministère opère un choix de conception économique dont les conséquences sont certainement encore mal mesurées. En introduisant dans la loi du 10 juillet 1915 la possibilité pour le syndicat d'intervenir sans avoir à justifier d'un préjudice subi par ses membres, la législation de l'époque, directement influencée par des penseurs comme Jean Jaurès lui-même, reconnaissait le fait qu'en matière salariale, le principal critère de justice était celui de l'indépendance des parties à la négociation. Longtemps réticente à cette conception de la négociation, la CGT, ou du moins une bonne part d'entre elle, s'y était ralliée et entendait ainsi faire des conventions collectives le moyen de l'amélioration de la condition ouvrière (36).

La publication de la série des arrêtés Parodi - et plus précisément encore celle des décisions de classification - est fondée sur une autre conception: en affinant les nomenclatures et les catégories, il deviendrait possible d'énoncer un ordre salarial global où la rémunération des individus est plus fondée sur le rang qui leur est reconnu que sur leur capacité - par l'action collective - à s'approprier légitimement une part de la richesse qu'ils contribuent à produire. C'est cette conception qui sera quelques années plus tard au fondement de la production et de la gestion des grilles salariales unifiées pour la fonction publique, conception dans laquelle, au moins formellement, la négociation avec les représentants des salariés n'est qu'une source seconde de la légitimité des règles salariales. Dans cette conception, la légitimité de la solution n'est plus fondée sur l'accord des parties adverses - comme c'est le cas dans une négociation-mais sur la connaissance des principes et des «lois» qui régissent l'ordre des choses. En soulignant ce clivage, on renforce l'hypothèse que l'opposition syndicale aux politiques salariales de ces années n'est pas, et de beaucoup, que le fait des syndicalistes inféodés au Parti communiste, dont l'opposition deviendrait forte lorsqu'ils constatent les difficultés du ministre à faire prévaloir les intérêts ouvriers (37).

La prévalence de cette conception homogène et unifiée de l'ordre salarial produit des bouleversements durables dans les institutions de relations professionnelles françaises. Et notamment en faisant prévaloir l'idée que le bon niveau de régulation des conditions de travail est le niveau national. Il était rare avant-guerre que des conventions collectives soient négociées à ce niveau; le plus souvent les textes existants se définissaient des champs d'application locaux ou régionaux. Les acteurs vont d'ailleurs manifester une certaine réticence à adopter ce changement: dans bien des branches,

(36) Ainsi Léon Jouhaux, en 1936, préfaçant la présentation de la loi sur les conventions collectives en vante clairement les mérites: «Les contrats collectifs constituent l'instrument le plus sûr pour soustraire la classe ouvrière à la dépendance abusive et à l'arbitraire patronal. Les contrats collectifs apportent un élément de sécurité et de stabilité dans la vie des travailleurs. Ils confèrent à la force de travail un caractère de dignité et de «respectabilité»... » (cité par Rolande Trempé, in Trempé, 1994, p.37).

(37) Pour un énoncé de cette position, $c f$. notamment LACROIXRIz A. (1983), «Un ministre communiste face à la question des salaires : l'action d'Ambroise Croizat de novembre 1945 à mai 1947 », Le Mouvement Social, 1983, 123, pp.3-44. 
la régulation régionale va reprendre ses lettres de noblesse, parfois avant même la loi de février 1950, comme dans la métallurgie où les textes signés par Ambroise Croizat en 1946 sont le plus souvent à portée régionale.

$*$
$* \quad *$

L'examen des textes permet d'identifier la persistance, au cours de l'année 1945, d'une politique fondée sur une administration centralisée des salaires. L'analyse plus fouillée nous a amenés à constater que cette utopie est largement combattue : les acteurs de terrain sont pour le moins sceptiques et le plus souvent réticents face à cette administration centralisée. Paradoxalement, l'identification des branches pour lesquelles l'ensemble des textes nécessaires à une bonne régulation salariale sont effectivement disponibles à la fin de l'année 1945 permet de se faire une idée de cette résistance. Ce sont en effet des secteurs dans lesquels il existait déjà une forte tradition de régulation, y compris conflictuelle, qui sont les premiers dans la liste des secteurs ou industries dans lesquels l'ensemble des textes de réglementation est publié le plus rapidement. La métallurgie, le BTP, la chimie dans les secteurs industriels, les assurances et le notariat dans les commerces et services sont des secteurs ou des industries dans lesquels existaient des conventions collectives avant la guerre et où la présence syndicale était notoire. Les instruments d'une régulation se sont donc mis en place le plus complètement dans les secteurs et les branches où existaient déjà une régulation de branche et une tradition de négociations. Qui plus est, comme on l'a vu sur la question de la gestion des salaires des employés dont la fonction est interprofessionnelle, ces secteurs ont recherché et obtenu que soient reconnues leurs spécificités et leur relative autonomie. Dans des secteurs où la régulation de branche existait déjà avant guerre notamment pour les ouvriers, elle a servi de support à la régulation gouvernementale des hiérarchies de salaires: les industries graphiques, le verre et le papier-carton sont de bons exemples pour illustrer cette situation. La régulation étatique est ainsi amenée à s'appuyer, voire à laisser la place, à la régulation de branche.

Des conventions collectives signées après 1936 aux arrêtés Parodi de 1945, la continuité est aisée à établir, y compris en passant bien souvent par la réglementation vichyste. Du moins si l'on privilégie les règles de fond et qu'on ne s'intéresse que secondairement aux niveaux de salaires. Pour une part donc, on peut estimer que la régulation Parodi des salaires ne fait que suivre les régulations de branches antérieurement en place. L'exemple du secteur des commerces alimentaires permet de poser l'hypo- thèse que, dans les secteurs où les régulations de branche étaient faibles, ce sont ces points d'accord locaux qui ont servi à étayer un canevas de classification qui dérivait des secteurs industriels mieux constitués.

Qui plus est, on peut encore constater que les régulations de branche ont servi de résistance aux innovations plus radicales proposées dans les arrêtés Parodi en matière de création de régulation interprofessionnelle. Cette réticence des autres acteurs, cumulée à la difficulté de négociation interne au gouvernement avec le ministère des Finances, conduira finalement le ministère à jeter l'éponge en matière de régulation salariale: du milieu de l'année 1946 à la crise de 1947, et a fortiori après celle-ci, le nombre de textes produits est minimal, et ce malgré l'inflation. Ce qui laisse supposer que la réglementation n'a plus d'effets réels sur les niveaux de salaires. On peut se demander si, de ce point de vue de la régulation salariale, la loi de février 1950 n'apparaît donc pas comme un aveu d'échec de la tentative gouvernementale.

Dans ces conditions, la bonne réputation qu'ont longtemps eue les arrêtés Parodi aux yeux des salariés et des syndicats semble tenir du paradoxe. Ils reposent sur une conception du fonctionnement des marchés du travail et de la légitimité des hiérarchies salariales alternative à la conception donnant le primat à la négociation de branche. En ce sens, cette conception de la hiérarchie salariale est conforme à la vision et aux valeurs partagées par les agents de l'État. Dont on peut penser qu'elle est celle qui régira l'ordre salarial français tel qu'il se mettra en place après la généralisation de la mensualisation.

La pertinence de l'analyse que nous venons de faire doit-elle être restreinte aux seuls domaines des salaires ou peut-on considérer qu'elle renseigne sur un aspect structurant du système français des relations professionnelles? On pourrait plaider la spécificité du domaine de la négociation salariale en remarquant que, dans un tel domaine, l'accord ne correspond que rarement à un véritable consensus entre les parties (SAGLIO, 2007). En d'autres termes, la constance de la revendication salariale rend l'accord difficile et la norme stable. L'intervention de l'État se justifie alors par l'incapacité de la formalisation de l'accord. L'histoire plus précise de l'année 1945 met à mal cette explication : car avant l'intervention de l'État pour s'approprier les règles de fond, les acteurs sont bien parvenus à négocier et à produire des accords. Ce serait donc alors plutôt la propension de l'État à s'arroger la fixation des règles de fond qui expliquerait la préférence des autres acteurs pour le non-engagement. L'inflation de la taille du Code du travail serait alors non seulement le signe, mais encore l'une des causes, de la difficulté de la négociation collective en France. 


\section{Bibliographie}

BEAu A.-S. (2001), Grand Bazar, modes d'emploi. Les salarié(es) d'un grand magasin lyonnais 1886-1974, thèse pour le doctorat d'histoire, université Lyon 2. (version en ligne).

BIERNACKI R. (1995), The fabrication of Labor, Germany and Britain, 1640-1914, University of California Press, 569 p.

Boltanski L. (1982), Les cadres. La formation d'un groupe social, Paris, Éditions de Minuit.

CAstoriadis C. (1975), L'institution imaginaire de la société, Paris, Éditions du Seuil.

Desrosières A., Thévenot L. (1988), Les catégories socioprofessionnelles, Paris, éditions La Découverte.

Duguit L. (1923), Traité de droit constitutionnel, deuxième édition, tome troisième «La théorie générale de l'État» (suite et fin) et tome cinquième et dernier «Les libertés publiques », Ancienne librairie Fontemoing et Cie, 1923 (t 1 à 4) et 1925 (t 5).

LARoque P. (1935), «Les conventions collectives de travail », rapport présenté par Monsieur Laroque, auditeur au Conseil d'État, Journal officiel du 3 janvier, annexe.

PiketTy T. (2001), Les hauts revenus en France. Inégalités et redistributions, 1901-1998, Paris, Grasset.

Reynaud J.-D. (1989), Les règles du jeu, l'action collective et la régulation, Paris, Armand Colin, (deuxième édition, 1993).

Saglio J. (1986), «Hiérarchies de salaires et négociations des classifications, France, 1900-1950», Paris, revue Travail et Emploi, $\mathrm{n}^{\circ} 27$.

Saglio J. (1987), «Les négociations de branche et l'unité du système français de relations professionnelles: le cas des négociations de classification », Paris, revue Droit Social, $\mathrm{n}^{\circ} 1$, pp.20-33.

SAglio (2007), «L'ordre salarial en France: la faiblesse du consensus, gage de stabilité», in Vatin F. (sous la direction de), Le salariat théorie, histoire et formes, La Dispute, pp.187-206.

Sellier F. (1961), Stratégie de la lutte sociale, Paris, Éditions Ouvrières, Éditions Économie et Humanisme.

Sellier F. (1984), La confrontation sociale en France, Paris, Presses universitaires de France.

Steinhouse A. (2001), Worker's Participation in PostLiberation France, Lexington books.

Terssac (de) G. (2003) (sous la direction de), La théorie de la régulation sociale de Jean-Daniel Reynaud, Débats et prolongements, Paris, éditions La Découverte.

Trempé R., (1994), «Les origines des conventions d'Arras », in Kourchid O., Trempé R. (1994), «Cent ans de conventions collectives, Arras, 1891-1991», Revue du Nord, hors série, $\mathrm{n}^{\circ} 8$, pp. 25-37.

\section{Annexe 1: le matériau analysé}

Les arrêtés et décisions concernant les rémunérations des salariés des entreprises privées ont été publiés au Journal officiel. La base de données utilisée a été constituée en identifiant les textes par trois moyens:

1) les tables des matières du Journal officiel publiées chaque mois au chapitre «Code du travail», sous la rubrique «salaires et indemnités». La série est cependant incomplète puisque de telles tables semblent n'avoir pas été publiées avant juin 1945, en septembre et en octobre. Qui plus est, même publiée, elle est parfois incomplète ;

2) une interrogation systématique de la base de données informatisée du Journal officiel, via le site Legifrance, en utilisant les mots clefs «arrêtés, décisions, salaires, appointements, traitements » permet de repérer les textes, mais non d'en disposer dans la mesure où la numérisation du Journal officiel sur ces années n'est pas achevée;

3) la lecture détaillée de chacun des textes permet d'identifier des textes complémentaires.

La base de données ainsi constituée pour la seule année 1945 comprend donc 126 textes d'arrêtés et 81 de décisions. On n'a pas comptabilisé les différents textes de rectification lorsqu'il s'agit de modifications mineures dont on ne parvient pas à la lecture à savoir s'il s'agit d'un changement de règle ou d'une rectification d'erreur matérielle dans la publication précédente. On n'a pas repris non plus les arrêtés et décisions dont l'objet porte sur les zones de salaires.

Une analyse plus rapide sur la période ultérieure, de janvier 1946 à mai 1947, a permis de valider certaines des hypothèses avancées. 